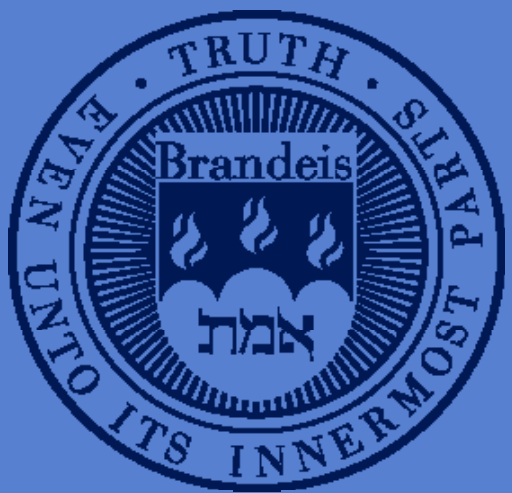

\title{
High-frequency Cash Flow Dynamics
}

Davide Pettenuzzo, Department of Economics, Brandeis University

Riccardo Sabbatucci, Stockholm School of Economics

Allan Timmermann, Rady School of Management, Univeristy of California San Diego

\section{Working Paper Series}




\title{
High-frequency Cash Flow Dynamics
}

\author{
Davide Pettenuzzo* \\ Brandeis University
}

\author{
Riccardo Sabbatucci ${ }^{\dagger}$ \\ Stockholm School of Economics
}

February 20, 2018

\author{
Allan Timmermann $\ddagger$ \\ UC San Diego
}

\begin{abstract}
We develop a new approach to modeling high-frequency dynamics in cash flows extracted from daily firm-level dividend announcements. Daily cash flow news follows a noisy process that is dominated by outliers so our approach decomposes this series into a persistent component, large but infrequent jumps, and temporary shocks with time-varying volatility. Empirically, we find that the persistent cash flow growth component is a better predictor of future dividend growth than alternative predictors from the literature. We also find strong evidence that news about the persistent cash flow component has a significantly positive effect on same-day stock market returns, while news about the temporary cash flow components has little effect on returns. Negative jumps in the cash flow process and higher cash flow volatility are associated with elevated stock market volatility and a higher probability of observing a jump in daily stock returns. These findings suggest that high-frequency news about the underlying cash flow growth process is an important driver not only of average stock market performance but also of the volatility and jump probability of stock prices.
\end{abstract}

Keywords: High-frequency cash flow news; predictability of dividend growth; jump risk; dynamics in stock returns; Bayesian modeling

*Department of Economics, Brandeis University. Email: dpettenu@brandeis.edu

${ }^{\dagger}$ Department of Finance, Stockholm School of Economics. Email: riccardo.sabbatucci@hhs.se

${ }^{\ddagger}$ Rady School of Management, UC San Diego. Email: atimmermann@ucsd.edu 


\section{Introduction}

On any given day, a multitude of firms typically announce cash flow news, but the number of firms, as well as the industries they belong to, can vary greatly over time. Such variation gives rise to a highly irregular cash flow news process and complicates investors' attempt to infer the underlying growth rate of cash flows for individual firms, industries, and for the economy as a whole. This is important because the resulting cash flow growth estimates play a key role in forecasting future cash flows, assessing cash flow risks, and valuing asset prices. ${ }^{1}$

While information extracted from firms' cash flow announcements is likely to be critical to understanding investors' cash flow expectations and, in turn, movements both in individual and aggregate stock prices, relatively few studies analyze predictability of cash flows and, in most cases, focus on quarterly or annual changes in aggregate dividends or earnings. ${ }^{2}$ However, cash flows that are aggregated in this manner conceal the rich dynamic patterns that arise in cash flows recorded at a higher frequency which reduces our ability to study important questions such as how strong and rapid cash flow growth responds to changes in the underlying state of the economy. ${ }^{3}$

Daily estimates of cash flows offer potentially large benefits to empirical tests of asset pricing models. A key challenge for such tests is that while high-frequency data are available on movements in individual and aggregate stock prices (e.g., daily or even intra-daily returns), cash flows of individual firms are observed at much lower frequencies (e.g., quarterly). The absence of high-frequency cash flow data reduces researchers' ability to estimate and test asset

\footnotetext{
${ }^{1}$ Savor and Wilson (2016) develop a learning model in which investors decompose cash flow news into firmspecific and market-wide components. Positive average covariances between the cash flow process of individual firms and of the broader market imply that bad (good) news on individual firms' cash flows are likely to result in reduced (increased) forecasts of aggregate cash flows. In turn, this cash flow learning channel implies that the stock returns of the announcing firms and of the aggregate stock market are positively correlated, justifying an "announcement risk premium" for exposure to individual firms' cash flows. Their model does not allow for lumpiness in cash flows ("jumps"), although in practice this is an important feature of earnings and dividend data.

${ }^{2}$ Cochrane (2008) finds little evidence of predictability of dividend growth, while van Binsbergen and Koijen (2010) and Kelly and Pruitt (2013) find some evidence that growth in dividends is predictable.

${ }^{3}$ To illustrate the loss in information from the common practice of aggregating cash flow news over the most recent 12-month period and updating this on, say, a monthly basis, suppose that firms' announcement dates are uniformly distributed across calendar dates. Every month when the cash flow estimate gets updated, the same weight is assigned to firms announcing cash flows close to the cutoff date and firms whose announcement date happened almost one year previously. This weighting automatically makes the resulting growth estimate stale and also introduces spurious serial correlation in the estimate - see, e.g., Working (1960).
} 
pricing models which rely on the joint dynamics of stock prices, expected returns and cash flow growth. For example, being limited to a smoothed annual dividend growth series means that we cannot explore to which extent daily return movements or stock market volatility are driven by cash flow news.

Several challenges complicate inference about daily cash flow dynamics. First, most firms' cash flows have a pronounced seasonal component related to weather patterns and holiday sales. Second, the number of firms announcing cash flow news on any given day can fluctuate between as little as zero firms to more than 200 firms and exhibits a clear pattern ("earnings season"). Third, the particular date on which a firm pays dividends or announces its quarterly earnings can vary widely from year to year, requiring that close attention be paid to constructing daily proxies that account for firm specific effects. Fourth, there is considerable heterogeneity across individual firms' cash flow processes. The combined effect of these factors is that daily news on cash flows tends to be very lumpy.

To address these challenges, in this paper we develop a new approach for extracting and modeling dynamics in high frequency (daily) cash flows. To handle firm-level seasonality we take a bottom-up approach that starts from changes in individual firms' dividends on a given day relative to their payments over the same quarter during the previous year. In contrast with conventional smoothed estimates, only data on those firms that announce dividend news on a given day are used to update the dividend growth estimate, thus ensuring that our measure is timely in picking up changes in the cash flow process. Moreover, by computing a dollar-weighted growth estimate, we account for variation in the size of the firms that pay dividends on any given day. ${ }^{4}$

To account for the lumpiness in daily values of year-on-year changes in firm-matched dividend growth, our modeling approach decomposes cash flows into a slowly evolving component that identifies time-variation in the mean of the cash flow process, a transitory component whose volatility is allowed to change over time, and large jumps whose probability of occurring can depend on the number of firms that announce dividends on a

\footnotetext{
${ }^{4}$ The daily horizon appears to be the highest frequency at which news on dividends can meaningfully be modeled; often, cash flow news are announced after the regular trading sessions in the stock markets have closed and so aggregating across firms that announced cash flows within a 24-hour interval - as opposed to modeling, say, hourly cash flow news - seems appropriate.
} 
given day. Empirically, all three components turn out to be important for capturing predictability in the dividend growth process and understanding the evolution in the uncertainty that surrounds growth in cash flows.

An important test of our approach is whether it can be used to generate more accurate forecasts of dividend growth than existing methods. Empirically, we find that the our estimate of the persistent dividend growth component is a strong predictor of future dividend growth while raw dividends do not have this property. Moreover, the predictive power of our approach compares favorably to alternative predictors of dividend growth computed using the filtering approaches of van Binsbergen and Koijen (2010) and Kelly and Pruitt (2013). We also find that our measure of the persistent dividend growth component is a positive and significant predictor of future growth in GDP and aggregate consumption. In sharp contrast, "raw" cash flow growth, or the individual jump or transitory shock components, are very noisy and turn out to have no predictive power over cash flow growth measured in the conventional manner.

Using our high frequency cash flow estimates, we next develop a model that allows us to estimate the effect of daily cash flow news on the mean, volatility and probability of a jump in stock market returns. We find that it is crucial to distinguish between different components of the cash flow process when analyzing the impact of cash flow news on stock prices. In particular, news about the persistent growth component has a large, positive and statistically significant effect on same-day stock returns, while news about jumps or shocks to the temporary cash flow component have a much smaller effect on mean stock returns. This finding does not rule out that these components of dividend growth have an effect on the dynamics of stock prices. In fact, we identify an "uncertainty effect" of dividend news on stock returns as negative jumps in dividend growth tend to increase both the volatility of stock market returns as well as the probability of observing a jump in stock returns. The latter effect is particularly large when few firms announce dividend news, i.e., on days with less cash flow news available to the markets. Positive jumps in cash flows have the reverse effect on stock market volatility and jump risk. Higher cash flow volatility also tend to spill over to higher volatility of stock market returns.

Our paper is related to a literature that attempts to estimate the effect of news on stock 
prices, especially on days with big market moves, see e.g., Cutler et al. (1989). ${ }^{5}$ A limitation of traditional event-study methods is that news stories are heterogeneous and based on qualitative information which makes it difficult to quantify the effect of each news story or compare the effect of different news over time. A second literature uses analyst expectations to gauge the news component from firms' earnings announcements, using the difference between actual and expected earnings as an estimate of the news. A limitation of this approach is that the estimated surprise is affected by biases in analyst estimates (e.g., Lim, 2001; Hong and Kubik, 2003) and by staleness in analysts' updates of their estimates which can contaminate consensus estimates. Our approach is fundamentally different as it uses actual cash flow data which, unlike analyst expectations, are not affected by biases in subjective estimates.

The methodology developed in our paper is related to that used by papers in the asset pricing literature which estimate models of stock return dynamics with stochastic volatility and jumps. However, to the best of our knowledge, no existing study has attempted to model the high-frequency dynamics in dividends using such methods, let alone estimate and test a model as general as ours. We are also not aware of any work that models the dependency of high-frequency dynamics in stock returns-e.g., time variation in the volatility and jump probability of returns-on cash flow news.

The outline for the paper is as follows. Section 2 introduces our data and explains how we construct a daily cash flow index from dividend announcement data. Section 3 explains our econometric modeling approach for dealing with jumps and a persistent (predictable) cash flow component and reports estimates of our model. Section 4 analyzes the extent to which our approach can be used to predict conventional measures of dividend growth. Section 5 develops a model relating dynamics in stock returns to cash flow news, while Section 6 presents results from a set of robustness tests, and Section 7 concludes.

\footnotetext{
${ }^{5}$ See also Andersen et al. (2007), McQueen and Roley (1993), and Boyd et al. (2005) for studies that look at the effect of news on movements in market prices.
} 


\section{Data}

This section explains how we construct our daily dividend growth series and describes the data sources that we use. Our analysis of daily cash flows focuses on growth in dividends which, as pointed out by Kelly and Pruitt (2013), has been the focus of a large asset pricing literature. ${ }^{6}$ Moreover, because earnings can be negative, defining growth in earnings poses challenges that are quite different from those arising when studying dividends.

The biggest effect of dividend news on asset prices is likely to come through their information content, so we focus on dividends as initially announced as opposed to the actual dividend payments. ${ }^{7}$ However, in Section 6.1.1 we also undertake an analysis of daily dividends viewed from the perspective of the payment date which allows us to compare the information effect to the direct cash flow effect from dividend payments.

\subsection{Sample Construction}

Our sample includes all ordinary cash dividends declared by firms with common stocks (share codes 10 and 11) listed on the NYSE-NASDAQ-AMEX from 1926 to 2016. ${ }^{8}$ We require firms to have valid stock prices and a valid figure for the number of shares outstanding when dividends are announced. Furthermore, we make sure there are no duplicate observations in the dataset and that each firm pays only one dividend at any point in time. ${ }^{9}$ Overall, our sample consists of 503,591 declared dividends. ${ }^{10}$

Corporate dividends have a strong firm specific component and also can display pronounced seasonal variation. Our analysis therefore computes dividend growth by comparing same-firm, same-(fiscal) quarter, year-on-year changes in cash flows. To this end, let $D_{y r, s}^{i}$ be the total dividends declared by firm $i$ on day $s$ in year $y r$, calculated as the

\footnotetext{
${ }^{6}$ See, e.g., Campbell and Shiller (1988), Cochrane (1992), Lettau and Ludvigson (2005), Koijen and Nieuwerburgh (2011), and Maio and Santa-Clara (2015).

${ }^{7}$ Announced dividends precede actual dividend payments by approximately 42 days, on average.

${ }^{8}$ Ordinary cash dividends have CRSP distribution codes below 2000.

${ }^{9}$ There are instances in CRSP in which a company declares or pays multiple dividends on the same day, using different distribution codes but still classified as ordinary dividends. We aggregate such dividends to convert them into a single dividend. As an example, on November 23rd 1983, PPL Corporation (permno 22517) declared two ordinary dividends of 39 and 21 cents.

${ }^{10}$ Following a recent update, CRSP no longer provides the dividend declaration date prior to 1962 and data until 1964 appear to be incomplete. Nonetheless, we also have an older version of the database in which the declared dividend dates start in 1926. As a consequence, we have 101,476 pre-1964 observations and 402,115 post-1964 observations.
} 
dividend per share times the number of outstanding shares. Moreover, let $I_{y r, s}^{i}$ be an indicator variable that equals one if company $i$ announces quarterly dividends on day $s$ in year $y r$, and otherwise takes a value of zero, while $\tilde{s}$ is the associated same-quarter, prior-year dividend announcement date for firm $i .{ }^{11}$ For example, company $i$ may have declared dividends on May 17, 2014 while it declared the corresponding quarter's prior-year dividends on May 9, 2013, in which case $s$ is May 17, 2014 and $\tilde{s}$ is May 9, 2013.

Using these notations, $N_{y r, s}=\sum_{i=1}^{N_{y r}} I_{y r, s}^{i}$ is the number of firms that announce dividends on day $s$ in year $y r$. Aggregating across firms, the total dollar value of dividends paid out on day $s$ in year $y r$ is $\sum_{i=1}^{N_{y r}} I_{y r, s}^{i} D_{y r, s}^{i}$. Similarly, the total value of dividends paid out by the same set of firms for the same fiscal quarter during the prior year is given by $\sum_{i=1}^{N_{y r}} I_{y r, s}^{i} D_{y r-1, \tilde{s}}^{i}$. Taking the ratio of these two numbers, we obtain a measure of the aggregate, year-on-year (gross) growth in dividends on day $s$ :

$$
D_{y r, s}=\frac{\sum_{i=1}^{N_{t}} I_{y r, s}^{i} D_{y r, s}^{i}}{\sum_{i=1}^{N_{t}} I_{y r, s}^{i} D_{y r-1, \tilde{s}}^{i}} .
$$

Note that the number of firms used in this calculation - as well as the identity of the specific firms - changes on a daily basis and from year to year as firms move their exact dividend announcement dates. Note also that only firms which satisfy that $I_{y r, s}^{i}=1$ are included in this calculation, ensuring that the same firms are used in both the numerator and denominator of the ratio. The definition in equation (1) accounts for seasonal components in dividends and uses the dollar amount paid in dividends by individual firms, implicitly applying value weights since large firms tend to have larger dividend payouts. Only seven percent of individual firms' year-on-year dividend growth observations in our sample are constant, suggesting that firms often change their dividends, even marginally, every year. ${ }^{12}$

\footnotetext{
${ }^{11}$ We use this notation to keep the exposition simple. More precisely, $\tilde{s}$ depends on both the firm $i$ and years $y r-1$ and $y r$, so that a more precise notation would be $\mathrm{s}(i, y r-1, y r)$.

${ }^{12} \mathrm{An}$ alternative approach that more explicitly accounts for heterogeneity in firm size is to first define individual firms' cash flow growth as

$$
y_{y r, s}^{i}=\left\{\begin{array}{ll}
\frac{D_{y r, s}^{i}}{D_{y r-1, \tilde{s}}^{i}} & \text { if } I_{y r, s}^{i}=1 \\
0 & \text { otherwise }
\end{array} .\right.
$$

In a second step we can use individual firms' market capitalization to aggregate the cash flow growth rates across firms that pay dividends on day $s$ in year $y r$ :
} 
As an illustration of these points, Figure 1 provides a plot of the number of firms, as well as the dollar dividend and the (net) growth rate from equation (1) during a single quarter (Q2 2014). The top panel shows substantial intra-quarter variation in the number of firms announcing dividends, consistent with the fact that firms tend to announce dividends around the same days. During this particular quarter, the maximum number of firms announcing dividends on any one day was 68 (on April 24), while the minimum number was zero (on June 22), and there were several days where more than 50 firms announced dividends.

The middle panel in Figure 1 shows the variation in the total value of dividends declared on any given date. This depends not only on the number of firms announcing dividends, but also on the size of the underlying companies because large firms tend to announce bigger dividends. ${ }^{13}$

Lastly, the bottom panel in Figure 1 shows the net daily dividend growth during the quarter. Peaks in this measure do not necessarily coincide with days where most firms announce dividends (top panel) or days in which the overall amount of dividends announced (middle panel) peaked. This is because the dividend growth rate depends on dividends announced by the same group of firms during the prior year as reflected in the denominator of equation (1). For example, the gross dividend growth rate on June 22 (1.15) is generated by a single firm announcing dividends on that day: the firm announced $\$ 155 \mathrm{~m}$ in dividends in Q2, 2014 and $\$ 135 \mathrm{~m}$ for Q2, 2013. The substantial variation in daily dividend growth rates that we observe reflects both heterogeneity across firms' dividend behavior and also variation in the number of firms announcing dividends on a given day.

An alternative to our bottom-up approach would be to extract dividends top-down from CRSP. Three limitations render this alternative approach unattractive. First, the CRSP index

$$
\begin{aligned}
y_{y r, s} & =\sum_{i=1}^{N_{y r}} I_{y r, s}^{i} \omega_{y r, s}^{i} y_{y r, s}^{i}, \quad \text { where } \\
\omega_{y r, s}^{i} & =\frac{M k t C a p_{y r, s}^{i} \times I_{y r, s}^{i}}{\sum_{i=1}^{N_{y r}} M k t C a p_{y r, s}^{i} I_{y r, s}^{i}}
\end{aligned}
$$

is the weight on company $i$ in the daily year-on-year value-weighted dividend growth calculation. By construction, $\sum_{i=1}^{N_{y r}} \omega_{y r, s}^{i}=1$ on all days in the sample. Results based on this alternative measure are very similar to those based on the measure in equation (1) and are, therefore, not reported here.

${ }^{13}$ The largest amount of dividends declared during Q2 2014, $\$ 7.12 \mathrm{bn}$, happened on April 24, while only $\$ 3.6 \mathrm{~m}$ of dividends were announced on June 30 . 
reflects the dividends that were distributed on a particular day but does not show when those dividends were announced. This distinction is crucial as firms typically announce dividends several days prior to the payment date and it is the news effect of announced dividends that we would expect to be important for movements in stock market returns and volatility. Second, the set of firms announcing or paying dividends on any given day is generally different from the set of firms announcing dividends on the same day one year earlier. As a consequence, year-on-year estimates of dividend growth from daily values of the CRSP index are difficult to interpret as they do not control for firm fixed effects. Third, the CRSP index contains many different assets such as ETFs and mutual funds (see Sabbatucci (2017)). Any dividend measure extracted top-down using the CRSP indexes is therefore not as clean as our measure which explicitly focuses on the actual nominal amount of dividends announced or distributed on any given day.

\section{$3 \quad$ Econometric model}

We propose a new measure of daily cash flow growth and it is worth studying its main features before introducing our formal modeling approach.

\subsection{Features of daily dividend growth}

Our data spans the period 1927-2016, but the first part of the sample is dominated by the Great Depression. For robustness, we therefore split the sample into halves and study both the full sample and the second half of the sample from 1973 to 2016 . Because dividends are non-negative, the measure in (1) is also non-negative and so we can compute the log change, $\Delta d_{y r, s}=\ln \left(D_{y r, s}\right)$. Figure 2 (top panel) plots $\Delta d_{y r, s}$ from 1973 to $2016 .{ }^{14}$ The daily dividend growth series is very spiky and is dominated by days with unusually large or small dividend growth. There is also evidence of a sustained decline in dividends during the financial crisis.

The features displayed by our daily series of year-on-year growth in dividends in Figure 2 can be summarized as follows: (i) the daily dividend growth series is very lumpy. This is due, in part, to variation in individual firms' cash flow growth, in part to changes in the composition of firms that, on any given day, announce their cash flows; (ii) daily dividend

\footnotetext{
${ }^{14}$ On days with no dividend announcements, we set the series to zero.
} 
news also appears to be driven by a persistent component which was particularly pronounced during the financial crisis of 2008/09; (iii) the volatility of daily cash flow news changes over time with unusually calm periods interchanged with more volatile periods.

These observations suggest that a model for daily news about cash flow growth must account for multiple components that display very different behavior. We accomplish this as follows. First, we account for lumpiness by allowing for a jump component in daily cash flow growth. Moreover, we allow the jump intensity to depend on the number of firms announcing dividends on a given day. Second, we incorporate a persistent component in the mean growth equation. Third, we account for time-varying volatility by modeling the volatility of the non-jump component of daily dividend growth as a stochastic volatility process.

This type of decomposition is not only of interest because it can better capture the dynamics in daily cash flow news. Most importantly, the decomposition is crucial for understanding and interpreting the effects of different types of cash flow news on movements in stock prices. For example, we would expect a change in the longer-lasting, persistent cash flow component to have a stronger effect on stock prices than a change in the transitory components.

We next introduce our econometric approach. To simplify notations, we use the daily indicator $t$ in place of the more cumbersome $y r, s$ notation used in equation (1). Thus, $\Delta d_{t}=\ln \left(D_{y r, s}\right)$ denotes the year-on-year growth in dividends on day $t$.

\subsection{A components model for daily dividend growth}

Our econometric model decomposes the daily dividend growth process into three parts, namely (i) a persistent term, $\mu_{d t+1}$, which captures a smoothly evolving mean component; (ii) a jump component, $\xi_{d t+1} J_{d t+1}$, where $J_{d t+1} \in\{0,1\}$ is a jump indicator that equals unity in case of a jump in dividends and otherwise is zero, while $\xi_{d t+1}$ measures the magnitude of the jump; (iii) a temporary cash flow shock, $\varepsilon_{d t+1}$, whose volatility is allowed to be persistent. Adding up these terms, we have

$$
\Delta d_{t+1}=\mu_{d t+1}+\xi_{d t+1} J_{d t+1}+\varepsilon_{d t+1} .
$$

We next introduce our assumptions on the individual components. We capture any 
persistence that may be present in the dividend growth process by assuming that $\mu_{d t+1}$ follows a mean-reverting first-order autoregressive process

$$
\mu_{d t+1}=\mu_{d}+\phi_{\mu}\left(\mu_{d t}-\mu_{d}\right)+\sigma_{\mu} \varepsilon_{\mu t+1}
$$

where $\left|\phi_{\mu}\right|<1$. The shocks $\varepsilon_{\mu t+1}$ are assumed to be normally distributed, $\varepsilon_{\mu t+1} \sim \mathcal{N}(0,1)$, and uncorrelated at all times with innovations to the temporary dividend shocks, $\varepsilon_{d t+1}$. When $\phi_{\mu}=0$, changes in the dividend growth rate process, $\Delta d_{t+1}$, become unpredictable and so this is a special case of our model.

Turning to the jump component, it turns out that there is a systematic relation between the probability of observing a jump in our daily cash flow series and the number of firms that announce dividends on a given day. In particular, days with few firms announcing news tend to have a higher chance of outliers in aggregate dividend growth, as the effect of diversifying outlier observations across multiple firms is smaller on such days. Accounting for this effect, we assume that the probability of a jump depends on the number of firms announcing their dividends on any given day. We capture this through a Probit model of the form

$$
\operatorname{Pr}\left(J_{d t+1}=1\right)=\Phi\left(\lambda_{1}+\lambda_{2} N_{d t+1}\right)
$$

where $N_{d t+1}$ denotes the number of firms announcing dividends on day $t+1$, while $\Phi$ stands for the CDF of a standard Normal distribution. The magnitude of the jumps is modeled as $\xi_{d t+1} \sim \mathcal{N}\left(0, \sigma_{\xi}^{2}\right)$

Finally, time-varying uncertainty about the temporary cash flow news component, $\varepsilon_{d t+1}$, is modeled by means of a stochastic volatility process:

$$
\varepsilon_{d t+1} \sim \mathcal{N}\left(0, e^{h_{d t+1}}\right)
$$

where $h_{t+1}$ is the log-variance of $\varepsilon_{d t+1}$ which is assumed to follow a mean-reverting process,

$$
h_{d t+1}=\mu_{h}+\phi_{h}\left(h_{d t}-\mu_{h}\right)+\sigma_{h} \varepsilon_{h t+1},
$$

where $\varepsilon_{h t+1} \sim \mathcal{N}(0,1)$ is uncorrelated at all times with both $\varepsilon_{d t+1}$ and $\varepsilon_{\mu t+1}$.

To summarize, our model accounts for a persistent mean-reverting component, 
time-varying volatility, and jumps. We evaluate the importance of the individual features of the model by comparing results from the general model in (2) to a simpler (no-jump) model that ignores both jump dynamics and stochastic volatility and so takes the form

$$
\Delta d_{t+1}=\mu_{d t+1}^{N J}+\varepsilon_{d t+1}
$$

where $\varepsilon_{d t+1} \sim \mathcal{N}\left(0, \sigma_{d}^{2}\right)$ and $\mu_{d t+1}^{N J}$ follows the process in equation (3). This comparison allows us to gauge the importance of incorporating jump dynamics and stochastic volatility.

\subsection{Estimation}

We adopt a Bayesian estimation approach that uses Gibbs sampling to estimate the model parameters. Details of our estimation procedure are provided in Appendix A while Appendix B documents the convergence properties of our estimation algorithm.

It is worth briefly describing the priors that underlie our model. We choose standard normal-gamma conjugate priors which simplify the process of drawing from the conditional distributions of the model parameters in the Gibbs samplers. Moreover, we specify independent priors for the parameters of both the mean, variance, and jump processes. As for the prior hyperparameters, for almost all of the parameters we use loose and mildly uninformative priors. The main exceptions are the persistence parameters, $\phi_{\mu}$ and $\phi_{h}$, whose priors we center on 0.99. Further details are provided in the appendices.

\subsection{Empirical estimates}

We next present estimates of the parameters of the econometric model introduced above. We also evaluate the empirical importance of the three components in the dividend growth process.

The middle and bottom panels in Figure 2 plot the persistent dividend growth component, $\mu_{d t}$, extracted from the daily dividend series shown in the top panel of the same figure using either the no-jump model (equation 7, middle panel) or the general jump model (equation 2,

bottom panel). The $\mu_{d t}^{N J}$ component extracted from the no-jump model evolves on the same scale as the daily dividend growth series from which it is extracted and thus, erroneously, assigns large daily spikes in the observed series to the persistent component, $\mu_{d t}$. In contrast, 
the jump model succeeds in separating the temporary spikes (noise) in the daily dividend series from the persistent component $\mu_{d t}$ which consequently is far smoother. Indeed, values of the persistent dividend growth component extracted from the general model fall on a far narrower scale than the unfiltered cash flow series, ranging from just below zero to 0.15 . As expected, the financial crisis in 2008-09 is associated with a notable drop in mean dividend growth which, for the only time in our sample turn negative, followed by a notable bounceback in the second half of 2009 and 2010.

Figure 3 provides details of the jump component obtained from our estimation procedure. The jump probability indicator, $J_{d t}$, in the top panel shows that the spikes in daily dividend growth is attributed mostly to jumps rather than to clusters with unusually high volatility from the transitory component, $\varepsilon_{d t}$, in equation (2). Moreover, on many days, the jump indicator variable is close to one. On such days we attribute, with a very high likelihood, much of the dividend growth shock to a jump. Jumps can be very large in magnitude, as shown in the bottom panel, which displays the estimated jump size, $\xi_{d t}$.

While Figures 2 and 3 show the evolution in the different dividend components, further insights can be gained by focusing on how our model decomposes the total variation in the dividend growth rate into temporary normal variation, jump, and mean-reverting components. Figure 4 performs this analysis for two days in our sample, namely December 8, 2008, in the middle of the global financial crisis, and August 5, 2010, i.e., during the recovery. The first day experienced a large negative shock to dividend growth. Our decomposition shows that this is attributed to small negative shocks to the persistent and transitory components and a large negative jump. Conversely, on August 5, 2010, the dividend growth news was small and positive which gets attributed to small positive realizations of the persistent component and the transitory shock and no jump.

Table 1 presents parameter estimates for our general dividend growth model in equations (2)-(6) for both the shorter sample (1973-2016) and the longer sample (1927-2016). We focus our discussion on the parameter estimates for the shorter sample but note that the estimates for the longer sample are very similar.

First consider the parameters determining the mean of the dividend growth process in equation (3). The long-run mean estimate $\mu_{d}=0.084$ corresponds to an $8 \%$ annualized 
nominal dividend growth rate. While quite high, this value is very close to the mean of the standard dividend growth measure (extracted from CRSP data) of $7.8 \%$ over the same sample period-well within the $90 \%$ credible set of $[0.064,0.104]$. The persistence parameter in the mean of the dividend growth process, $\phi_{\mu}$, has a mean of 0.998 and a narrow $90 \%$ credible set that ranges from 0.997 to 0.999 . While highly persistent, shocks to the mean process (3) are very small as shown by the estimated $\sigma_{\mu}=0.002$. Our model thus identifies a small, but highly persistent component in the dividend growth process.

The stochastic volatility process in equation (6) is also quite persistent as evidenced by the estimate of $\phi_{h}$ whose mean is 0.963 with a standard deviation of 0.002 . The jump intensity parameters $\left(\lambda_{1}\right.$ and $\left.\lambda_{2}\right)$ have mean values of -1.59 and -0.025 , respectively, and are accurately estimated. These parameter estimates imply that a jump occurs every sixty days on average and the negative and highly significant estimate of $\lambda_{2}$ show that the jump probability tends to be lower on days where a large number of firms announce dividends.

To give a sense of how sensitive the dividend growth jump probability is to the number of firms announcing dividends on a given day, $N_{t}$, Figure 5 plots the jump intensities for three values of $N$ chosen to match the $25 \mathrm{th}$, median and 75 th percentiles of the distribution of the daily number of announcing firms. On days with a large number of announcing firms (75th percentile, or 36 firms on average), the jump intensity distribution is centered on a number a little over 0.005 , corresponding to a jump on average every 200 days. On days with a typical number of announcing firms (median, or 22 firms), the jump intensity is centered around its average value near 0.016, implying a jump every 60 days. Finally, on days with a small number of announcing firms (25th percentile, or 12 firms), the probability of a jump is centered just below 0.03 , corresponding to a jump every 35 days.

The estimated standard deviation of the jump size $\left(\sigma_{\xi}\right)$ has a mean of 1.43 , suggesting that jumps in daily dividend growth, though relatively infrequent, can be very large when they do occur. This value can be compared to the estimated mean of $\sigma_{h}$ which, at 0.20 , is seven times smaller. In other words, shocks to daily dividend growth process coming from the jump component tend to be seven times bigger than the regularly occurring $\varepsilon_{d t}$ shocks. 


\section{Predictability of dividend growth}

Predictability of dividend growth has featured prominently in discussions of asset pricing models. Cochrane (2008) finds little evidence of predictability of US dividends, while studies such as van Binsbergen and Koijen (2010) and Kelly and Pruitt (2013) argue that dividend growth is, to some extent, predictable. ${ }^{15}$ The parameter estimates from our dividend model in Section 3.4 show that the daily dividend growth process contains a small, but highly persistent component which, in principle, should be able to pick up dividend growth predictability. This section explores the implications of our model estimates for predictability in dividend growth.

Existing studies on dividend growth predictability use time-aggregated dividends measured over longer horizons than our daily interval. To explore the extent to which our estimate of the persistent dividend growth component is capable of predicting dividends as conventionally measured-and to make our results directly comparable to existing ones-we construct quarterly and annual measures of dividend growth from the CRSP index with and without dividends. ${ }^{16}$

\subsection{Correlation with conventional dividend growth}

To assess the contemporaneous relation between the conventional measure of dividend growth, $\Delta d_{t}^{C R S P}$, and our estimate, we regress $\Delta d_{t}^{C R S P}$ on the persistent dividend growth component obtained from our jump model, $\mu_{d t}$, measured at the end of period $t$ :

$$
\Delta d_{t}^{C R S P}=\alpha+\beta \mu_{d t}+\varepsilon_{t}
$$

Panel A of Table 2 reports results at the annual and quarterly (4Q-rolling) frequencies-the frequencies most commonly used in the literature to account for dividend seasonality. First consider the findings for the sample period that starts in 1973 . At the quarterly horizon we find a strong positive contemporaneous correlation between $\mu_{d t}$ and the

\footnotetext{
${ }^{15} \mathrm{~A}$ recent literature uses dividend futures to estimate the term structure of dividends. In particular, van Binsbergen et al. (2012) and van Binsbergen and Koijen (2016) present evidence on the term structure of the equity premium, recovering prices of dividend strips and show that their expected returns are higher than those on the underlying index. Kragt et al. (2015) estimate a model for the term structure of discounted risk-adjusted dividend growth using dividend derivatives for four major stock markets.

${ }^{16}$ Most researchers extract aggregate dividends, $D_{t}$, from CRSP as the difference between the cum-dividend return (VWRETD), $R_{t}^{\text {cum }}$, minus the ex-dividend return (VWRETX), $R_{t}^{e x}$, multiplied by the previous exdividend index level, $P_{t-1}^{e x}$, i.e., $D_{t}=\left(R_{t}^{c u m}-R_{t}^{e x}\right) \times P_{t-1}^{e x}$. Using the resulting aggregate dividend series, the $\log$ dividend growth rate can be computed as $\Delta d_{t}^{C R S P}=\ln \left(\frac{D_{t}}{D_{t-1}}\right)$.
} 
conventional dividend growth measure as reflected in an $R^{2}$ value of $28 \%$ and a t-statistic of 6.93. In the longer sample that starts in 1927, the persistent dividend growth component continues to be strongly correlated with the contemporaneous growth in dividends measured in the conventional manner, generating an $R^{2}$ value of $35 \%$ and a t-statistic above seven.

At the annual horizon we continue to find that our estimate of the persistent dividend growth component, $\mu_{d t}$, is significantly correlated with contemporaneous dividend growth although the $R^{2}$ values, at $10 \%$ and $15 \%$, respectively, are somewhat lower than at the quarterly horizon.

\subsection{Predictive regressions}

Next, we estimate predictive regressions that regress next-period dividend growth on the current persistent dividend component, $\mu_{d t}$, the current log dividend-price ratio extracted from CRSP, $d p_{t}$, and current and lagged dividend growth:

$$
\Delta d_{t+1}^{C R S P}=\alpha+\beta \mu_{d t}+\gamma d p_{t}+\sum_{j=1}^{3} \rho_{j} \Delta d_{t+1-j}^{C R S P}+\varepsilon_{t+1} .
$$

We include the log dividend-price ratio in this regression because this has been suggested as a predictor of cash flow growth in a variety of studies (e.g., Cochrane (1992), Cochrane (2008), Lettau and Nieuwerburgh (2008), and Cochrane (2011)).

Panel B of Table 2 shows that the persistent component of dividend growth, $\mu_{d t}$, has strong predictive power over future dividend growth recorded at the quarterly horizon. In the shorter post-1973 sample, the lagged persistent dividend growth component obtains a t-statistic of 4.5 after accounting for the effect of lagged dividend growth and the lagged dividend-price ratio. Moreover, at 0.28 the $R^{2}$ remains as high as it was in the contemporaneous regression. Again, this finding is not sensitive to the sample period. Starting the sample in 1927, the coefficient on $\mu_{d t}$ obtains a t-statistic of 4.3 and the predictive regression has an $R^{2}$ value of 0.41. Interestingly, the coefficient on the lagged dividend-price ratio is not significant in any of these regressions, while the first two lags of the lagged dividend growth are significant in some of the models, but not always with the expected (positive) sign.

The predictive power of $\mu_{d t}$ over future dividend growth is somewhat weaker at the annual 
than at the quarterly horizon. This is perhaps not surprising in light of the mean reversion in $\mu_{d t}$ which reduces the predictive power of this component at long horizons. Still, we continue to find at the annual horizon that the statistical significance of our dividend growth measure is robust to the inclusion of lagged dividend growth and the dividend-price ratio in the predictive regression.

\subsection{Alternative predictors of dividend growth}

Our study is not the first to use filtering methods to obtain an estimate of dividend growth. For example, van Binsbergen and Koijen (2010) use a latent variables approach to estimate a loglinearized present value model consisting of expected returns and expected dividend growth rates for the aggregate stock market. Because the expected values of dividend growth rates are unobserved, van Binsbergen and Koijen (2010) use Kalman filtering methods to extract the underlying series and generate forecasts of cash flows. Empirically, van Binsbergen and Koijen (2010) find that annual dividend growth rates are less persistent, but more predictable, than stock returns.

Kelly and Pruitt (2013) assume that individual firms' stock returns and log cash flow growth rates are a linear function of a set of unobserved common factors which can be estimated using a three-pass regression (partial least squares) methodology. In turn, cash flow growth can be projected on the common factors to generate a dividend growth forecast. Empirically, Kelly and Pruitt find strong in-sample evidence of annual cash flow growth predictability. Their out-of-sample results are somewhat mixed; in the Depression-era from 1930-1940, dividend growth appears to be highly unstable and hard to predict while conversely out-of-sample predictability is stronger over the sample 1940-2010.

We next compare our dividend growth estimates to results based on the approaches of van Binsbergen and Koijen (2010) and Kelly and Pruitt (2013). ${ }^{17}$ To this end, the top panel in Figure 6 plots realized values of quarterly dividend growth against the persistent growth component estimated from our model, $\mu_{d t}$, (sampled annually) and the van Binsbergen and Koijen (2010) measure, $g_{t}^{V B K}$. The bottom panel repeats the exercise, plotting monthly

\footnotetext{
${ }^{17}$ We are grateful to Seth Pruitt for sharing data and computer code which allowed us to replicate the results in Kelly and Pruitt (2013).
} 
dividend growth against our persistent dividend growth series, $\mu_{d t}$, (sampled monthly) and the Kelly and Pruitt (2013) estimate, $g_{t}^{K P}$.While the different dividend growth estimates are clearly correlated, there are also some notable differences. For example, our persistent dividend growth measure shows a sharper decline during the global financial crisis compared with the two alternative estimates.

To conduct a more formal comparison, Panel $\mathrm{C}$ in Table 2 reports results from regressions of the observed future dividend growth on the growth estimate implied by the three approaches we are comparing. Note that findings from the two approaches are not directly comparable as van Binsbergen and Koijen (2010) study cash-reinvested, annual dividend growth while Kelly and Pruitt (2013) use monthly dividend growth extracted from CRSP. We therefore report separate results for the annual and monthly frequencies used in the two studies.

In the univariate regressions, all three growth estimates clearly have predictive power over future dividends. For example, the growth estimate of van Binsbergen and Koijen (2010) obtains a t-statistic of 2.94 and generates an $R^{2}$ value of $14 \%$ in the annual sample from 1946 to 2015. For comparison, the t-statistic on our $\mu_{d t}$ estimate is 5.77 and the associated $R^{2}$ value is $39 \%$. Including both the $\mu_{d t}$ and $g_{t}^{V B K}$ measures in the regression, we obtain a very large t-statistic on $\mu_{d t}$ (6.03), while the t-statistic on the estimate of van Binsbergen and Koijen (2010) drops to 2.03. The $R^{2}$ value of this regression is $44 \%$. This is notably higher than the value obtained when only $g_{t}^{V B K}$ is used as a predictor, thus demonstrating the extra predictive power possessed by our estimate of the persistent growth component.

In monthly dividend growth regressions from 1940 to 2016, the growth estimate of Kelly and Pruitt (2013) generates a t-statistic of 4.86 and an $R^{2}$ value of $13 \%$. For comparison, the t-statistic obtained when instead we use our $\mu_{d t}$ component is 9.96 and the $R^{2}$ value is $32 \%$. Including both $\mu_{d t}$ and $g_{t}^{K P}$ as predictors in the regression, $\mu_{d t}$ obtains a t-statistic of 8.09 while the t-statistic of the growth estimate of Kelly and Pruitt (2013) declines to 2.06. Moreover, the $R^{2}$ value of this regression is $33 \%$ which is marginally higher than the value from the regression only on $\mu_{d t}$, though notably higher than the $R^{2}$ value from the univariate dividend regression on $g_{t}^{K P}$.

These results show that the persistent component in dividend growth extracted from daily dividend announcements possesses strong predictive power over actual dividend growth at 
both the monthly and annual frequencies. Moreover, our estimate adds substantial predictive power to existing dividend growth estimates.

To formally test and compare the predictive power of the three dividend growth estimates, we run a series of forecast encompassing regressions:

$$
\begin{aligned}
& \Delta d_{t+1}^{C R S P}=\alpha+\beta_{1} \mu_{d t+1}+\left(1-\beta_{1}\right) g_{t}^{V B K}+\varepsilon_{t+1}, \\
& \Delta d_{t+1}^{C R S P}=\alpha+\beta_{1} \mu_{d t+1}+\left(1-\beta_{1}\right) g_{t}^{K P}+\varepsilon_{t+1} .
\end{aligned}
$$

The larger is $\beta_{1}$ in these regressions, the greater the weight on our dividend growth estimate and the smaller the weight on the competing model estimate. In particular, a value of $\beta_{1}=1$ suggests that $\mu_{d t}$ dominates (encompasses) either $g_{t}^{V B K}$ (top regression) or $g_{t}^{K P}$ (bottom regression). ${ }^{18}$

The bottom two rows of Table 2 show that the estimate of $\beta_{1}$ in the encompassing regression that includes $\mu_{d t}$ and the van Binsbergen and Koijen (2010) dividend growth estimate equals 0.81 , so that the persistent dividend growth estimate from our model obtains a weight of $81 \%$ while the weight on the van Binsbergen and Koijen (2010) estimate equals $19 \%$. Moreover, the estimated weight on $\mu_{d t}$ is statistically significant at the $1 \%$ level while the weight on the van Binsbergen and Koijen (2010) estimate is significant at the $10 \%$ level. Very similar results are obtained from the second regression. Here the weight on $\mu_{d t}$ is $77 \%$, while the weight on the Kelly and Pruitt (2013) dividend growth estimate is 23\%, with both being significant at the $1 \%$ level. Thus, while the dividend growth estimates of van Binsbergen and Koijen (2010) and Kelly and Pruitt (2013) contain information relevant for predicting future dividend growth over and above the information in our $\mu_{d t}$ estimate, these regressions show that our dividend growth estimate performs very well compared to existing state-of-the-art alternatives.

\subsection{Cash flow news and economic activity}

We next examine the relation between our estimate of the persistent component of dividend growth news and two measures of macroeconomic growth, namely GDP and consumption growth, both of which have been examined by authors such as Liew and Vassalou (2000) and

\footnotetext{
${ }^{18}$ Note that $g_{t}=E_{t} \Delta d_{t+1}$ is the forecast of next period dividend growth.
} 
Bansal and Yaron (2004)). ${ }^{19}$ Figure 7 plots quarterly GDP and consumption growth against our $\mu_{d t}$ measure sampled quarterly. We observe a clear and positive relation between the persistent dividend growth component on the one hand and consumption and GDP growth on the other.

To obtain formal results on the relation between the three measures, we estimate the following quarterly contemporaneous regression

$$
\Delta y_{t}=\alpha+\beta \mu_{d t}+\varepsilon_{t}
$$

where $\Delta y_{t}$ is either the (log) GDP or consumption growth. Results are reported in Panel A of Table 3. Our estimate of the persistent component of dividend growth, $\mu_{d t}$, is positively related to contemporaneous growth in both GDP and consumption, with statistically significant coefficients of .14 and .13, respectively, and t-statistics around six. The $R^{2}$ values of the regressions are $26 \%$ and $30 \%$, respectively, suggesting that our measure picks up more than a quarter of the variation in these macroeconomic variables.

We also consider predictive regressions of the form

$$
\Delta y_{t+1}=\alpha+\beta_{1} \mu_{d t}+\beta_{2} \Delta y_{t}+\varepsilon_{t+1}
$$

where we include one lag of the dependent variable, $\Delta y_{t}$, to control for persistence in consumption or GDP growth. Panel B in Table 3 reports the results from the regression in (12). In the univariate regressions, our persistent dividend growth measure, $\mu_{d t}$, generates positive coefficients of 0.14 and 0.13 with t-statistics of 4.57 and 4.72 for GDP growth and consumption growth, respectively. Moreover, with $R^{2}$ values of $21 \%$ and $26 \%, \mu_{d t}$ clearly has strong predictive power over future GDP and consumption growth.

We conclude from this evidence that our persistent cash flow measure $\mu_{d t}$ helps explain and predict variation in macroeconomic growth. This is consistent with our earlier finding that $\mu_{d t}$ predicts future dividend growth and shows that the earlier result carries over to broader measures of economic growth.

\footnotetext{
${ }^{19}$ The Gross Domestic Product series is downloaded from FRED and is seasonally adjusted, see https: //fred.stlouisfed.org/series/GDP. Consumption expenditures are the sum of non durable consumption plus services from Table 2.3.5 of the National Income and Product Accounts (NIPAs) and are available on the Bureau of Economic Analysis (BEA) website.
} 


\subsection{Relation to other measures of economic and financial activity}

Since our daily cash flow growth measure reflects general macroeconomic conditions, it can be viewed as an economic indicator similar to existing measures such as the macroeconomic uncertainty measure of Jurado et al. (2015), the economic policy uncertainty measure of Baker et al. (2016), the ADS business conditions index of Aruoba et al. (2009), the credit spread indicator of Gilchrist and Zakrajek (2012), and "noise" in the Treasury market (Hu et al. (2013)). ${ }^{20}$ Previous research has addressed whether these measures can be used to improve forecasting and evaluation of the state of the economy in real time, especially during recessions and financial crises. Similarly, our high-frequency cash flow measure can be used to evaluate the "financial soundness" of the economy by understanding its relationship with some of these proxies. $^{21}$

Panel A of Table 4 shows estimates of the correlations between the persistent dividend component $\mu_{d t}$ and some of these daily measures of financial and macroeconomic conditions. Our persistent dividend growth measure has a highly significant negative correlation of 0.53 with the VIX, suggesting that dividend growth is lower in times with high uncertainty, which tends to coincide with economic recessions. Confirming this finding, $\mu_{d t}$ also has a significantly negative correlation of -0.23 with the policy uncertainty index of Baker et al. (2016) and a negative correlation of -0.59 with the liquidity noise index of $\mathrm{Hu}$ et al. (2013), indicating that firm payouts are lower in times with greater uncertainty. Finally, our cash flow index is positively correlated with the ADS index of Aruoba et al. (2009), obtaining a highly significant correlation of 0.32 , and with the daily inflation index of Cavallo and Rigobon (2016) (correlation of 0.78). These findings show that our persistent dividend growth measure is significantly negatively correlated with risk proxies, e.g., stock market volatility and policy uncertainty, but positively correlated with economic growth and inflation.

Panel A uses levels and so the correlation estimates described above are driven by

\footnotetext{
${ }^{20}$ Aruoba et al. (2009) measure economic activity at the daily frequency using a variety of stock and flow data observed at mixed frequencies. Their approach extracts the state of the business cycle from a latent factor that affects all observed variables. Jurado et al. (2015) provide econometric estimates of time-varying macroeconomic uncertainty and show that important uncertainty episodes appear far more infrequently than indicated by popular uncertainty proxies. However, when such episides do occur, they tend to be larger, more persistent, and more correlated with real economic activity.

${ }^{21}$ Other proxies include the aggregate external cost of financing (Eisfeldt and Muir (2016)) and bank-loan supply conditions (Becker and Ivashina (2014)) which are related to firms' dividend payout policy.
} 
common, persistent factors reflecting the state of the economy. Panel B sheds light on short-run correlations by reporting the correlations between daily changes in the underlying indexes. Changes in our daily dividend growth index are significantly positively correlated both with changes in the ADS index and changes in daily inflation, suggesting that our measure in part captures fundamental information reflected in other macroeconomic variables.

\section{Return dynamics and cash flow news}

A key motivation for our new decomposition of cash flow news is that it can shed light on the drivers of the dynamics in daily stock prices. From a theoretical perspective, we would expect the three components to have very different impact on stock prices. For example, we would expect a purely temporary shock to the cash flow process $\left(\varepsilon_{d t}\right)$ to have very little effect on stock prices, whereas a shock to the persistent dividend growth component $\left(\mu_{d t}\right)$ should have a larger impact. Similarly, shocks to the volatility of cash flows might influence the mean and volatility of aggregate stock market returns, as investors attempt to learn about the underlying cash flow process, and hence affect returns through a risk premium channel. Documenting the importance of these effects is important as the sources of daily movements in stock prices are poorly understood.

To address these points, in this section we use our daily dividend growth estimates to conduct an analysis of the relation between stock market returns and news about the dividend growth process. We first develop a new dynamic model that is sufficiently flexible to allow the distribution of stock market returns to incorporate cash flow news. We then develop a set of key hypotheses linking movements in stock market returns to our estimates of cash flow dynamics. Finally, we report estimates of our return model and results from empirical tests of the hypotheses.

\subsection{Stock returns and cash flow dynamics}

A long-standing debate in the asset pricing literature is concerned with how important time variation in expected cash flows is to explaining variation in stock market returns. ${ }^{22}$ Some

\footnotetext{
${ }^{22}$ See, e.g., Cochrane (2008) and van Binsbergen et al. (2012).
} 
studies argue that growth in cash flows is largely unpredictable. If dividends follow a random walk with a drift and dividend growth is not predictable, time variation in risk premia become more important to explaining movements in stock returns. Conversely, variation in the predictable component of dividend growth should impact stock prices by more than shocks to temporary components of dividend growth. Our model allows us to easily update and compute forecasts of future cash flows and so can readily be used to estimate the importance of time variation in cash flow expectations.

To analyze the effect of dividend news on stock returns, we develop a novel dynamic model for daily stock returns. As in earlier studies such as Eraker et al. (2003), we allow for stochastic volatility effects and jumps in stock returns, but our model generalizes existing approaches by linking stock market volatility and jumps to the corresponding dynamic components in the cash flow process. We accomplish this using a two-stage approach that first estimates the dividend growth rate model, then includes the extracted components in the model for stock market returns.

Our approach takes advantage of the timing of firms' dividend announcement and movements in stock returns. Firms generally determine their dividends several days prior to observing the aggregate returns on the day of the dividend announcement. Given this timing, we can treat the estimated dividend components as being pre-determined relative to aggregate stock market returns.

Our model for the dynamics in daily stock market returns takes the following form:

$$
r_{t+1}=\mu_{r t+1}+\xi_{r t+1} J_{r t+1}+\beta_{1} \Delta \mu_{d t+1}+\beta_{2} \exp \left(h_{d t+1} / 2\right)+\beta_{3} \xi_{d t+1} J_{d t+1}+\beta_{4} \varepsilon_{d t+1}+\varepsilon_{r t+1} .
$$

Analogously with the dividend model, $\mu_{r t+1}$ captures a persistent component in returns, $\xi_{r t+1} J_{r t+1}$ represent jumps in returns with $J_{r t+1} \in\{0,1\}$ being a jump indicator and $\xi_{r t+1}$ measuring the magnitude of a jump, while $\varepsilon_{r t+1} \sim \mathcal{N}\left(0, e^{h_{r t+1}}\right)$ is a diffusion term with timevarying log-volatility $h_{r t+1}$. The four additional components, $\beta_{1} \Delta \mu_{d t+1}, \beta_{2} \exp \left(h_{d t+1} / 2\right)$, $\beta_{3} \xi_{d t+1} J_{d t+1}$, and $\beta_{4} \varepsilon_{d t+1}$ capture spillover effects on returns from the conditional mean, conditional volatility, jump, and diffusion components of the dividend growth process. We discuss the economic interpretation of these terms below. 
The mean of the return process, $\mu_{r t+1}$, is assumed to follow a mean-reverting $\operatorname{AR}(1)$ process:

$$
\mu_{r t+1}=\mu_{r}+\phi_{\mu r}\left(\mu_{r t}-\mu_{r}\right)+\sigma_{\mu r} \varepsilon_{r \mu t+1}, \quad \varepsilon_{r \mu t+1} \sim \mathcal{N}(0,1)
$$

where $\varepsilon_{r \mu t+1}$ is assumed to be uncorrelated at all times with the innovation in the temporary return component, $\varepsilon_{r t+1}$ and $\left|\phi_{\mu r}\right|<1$.

The $\log$ variance of $\varepsilon_{r t+1}$ is also assumed to evolve according to a mean-reverting, autoregressive process modified to include the volatility and jump components extracted from the dividend process:

$$
h_{r t+1}=\mu_{h r}+\phi_{h r}\left(h_{r t}-\mu_{h r}\right)+\gamma_{1} \Delta \mu_{d t+1}+\gamma_{2} h_{d t+1}+\gamma_{3} \xi_{d t+1} J_{d t+1}+\sigma_{h r} \varepsilon_{r h t+1},
$$

where $\varepsilon_{r h t+1} \sim \mathcal{N}(0,1)$ is uncorrelated at all times with both $\varepsilon_{r t+1}$ and $\varepsilon_{r \mu t+1}$.

Finally, we allow the jump intensity of returns to depend on the number of firms announcing dividends on any given day, $N_{t}$, as well as on the jumps in the dividend growth process:

$$
\operatorname{Pr}\left(J_{r t+1}=1\right)=\Phi\left(\lambda_{1}^{r}+\lambda_{2}^{r} N_{d t+1}+\lambda_{3}^{r}\left(\xi_{d t+1} J_{d t+1}\right)\right) .
$$

The magnitude of the jump, $\xi_{r t+1}$, is modeled as $\xi_{r t+1} \sim \mathcal{N}\left(0, \sigma_{\xi_{r}}^{2}\right)$.

Our return model can be compared to specifications adopted in previous studies in the asset pricing literature such as Chib et al. (2002) and Eraker et al. (2003). Chib et al. (2002) model daily returns on the $\mathrm{S} \& \mathrm{P} 500$ index using an additive jump process in the return equation of a discrete time stochastic volatility (SV) model, while Eraker et al. (2003) compare several SV models with additive jump components in both the return and variance equations applied to daily returns on the S\&P 500 and Nasdaq indexes. Eraker et al. (2003) find that allowing for jumps in both the mean and the variance processes generate quite different price dynamic compared to a strategy of adding diffusion factors or only allowing for jumps in returns. ${ }^{23}$

There are several key differences between our specification and the models used in earlier studies. First, and most importantly, we include the components extracted from the daily

\footnotetext{
${ }^{23}$ Chan and Grant (2016a,b) discuss and compare various SV models that are widely used in the literature to model financial and macroeconomic time series with and without jumps in the mean equation, and outline efficient algorithms for fitting these models that build on fast band matrix routines.
} 
dividend growth process in the specification of mean returns dynamics (13). Second, we allow for a mean reverting component, $\mu_{r t}$, in stock returns. Third, we allow the volatility of stock market returns to be affected by both the volatility and jumps of the dividend growth process, (15). Finally, the jump probability of returns in our model can depend not only on the number of firms announcing dividends on a given day but also on jumps in news about dividend growth, (16). These are features that have not previously been explored when modeling stock returns.

\subsection{Hypotheses}

We next develop a set of economic hypotheses that we use to guide our empirical analysis. Note that the direction of causality is well-determined in our setting: It is highly unlikely that the dividends announced by firms on any given day could be affected by stock returns on that day as corporate boards meet to determine dividend payments well in advance of the day where they get announced. Conversely, stock prices are expected to react quickly to cash flow news announcements.

Our first hypothesis is that news about the permanent component of cash flows, $\Delta \mu_{d t+1}$, should have a significantly positive and larger effect on same-day stock returns than a shock to the transitory cash flow component, $\varepsilon_{d t+1}$, or jumps in the cash flow process, $\xi_{d t+1} J_{d t+1}$. These observations translate into the following hypothesis about the parameters in equation (13):

Hypothesis 1. Stock returns tend to be higher on days with positive news about the persistent growth component of cash flows, while temporary shocks to cash flows should not have any effect on stock returns.

$$
H_{1}: \beta_{1}>0 \text { and } \beta_{3}=\beta_{4}=0 \text {. }
$$

Our second hypothesis is that higher cash flow volatility is associated with a positive risk premium as it indicates an environment with higher uncertainty about fundamental growth. We formulate this hypothesis as a statement about the effect of $\exp \left(h_{d t+1} / 2\right)$ on stock returns, noting that this term will be dominated by variation in the conditional variance of the $\varepsilon_{d t+1}$ 
cash flow component due to the high persistence in the $h_{d t+1}$ process.

Hypothesis 2. Stock returns tend to be higher in periods with high cash flow volatility and greater uncertainty about cash flow growth.

$$
H_{2}: \beta_{2}>0
$$

Our third hypothesis is that higher cash flow volatility and jumps in cash flows are drivers of the return volatility process. Specifically, we would expect positive news about $\Delta \mu_{d t+1}$ to be associated with lower return volatility as it indicates stronger fundamentals which are traditionally associated with a less uncertain environment. Conversely, we expect higher cash flow volatility and large negative jumps to be associated with more volatile stock returns as they indicate greater uncertainty about cash flows and worse growth prospects. In the context of the return volatility equation (15), this suggests the following hypothesis:

Hypothesis 3. Return volatility tends to be higher on days with negative news about the persistent growth rate, higher cash flow volatility, and negative jumps in cash flows:

$$
H_{3}: \gamma_{1}<0, \gamma_{2}>0 \text { and } \gamma_{3}<0
$$

Fourth and finally, we expect that jumps in cash flows will increase the probability of observing same-day jumps in stock returns. Jumps in cash flows increase uncertainty about the prospects for stock prices and make it more likely to see large movements in these. Moreover, in the same way that negative news increase stock market volatility more than positive news through a leverage effect, we would expect a negative jump, i.e., a sharp downward adjustment in the cash flow process, to be associated with a particularly high chance of observing a jump in returns. Days with fewer signals about fundamentals, i.e., days with fewer firms announcing their dividends, can also be expected to be more uncertain, increasing the chance of observing a jump in stock returns. Using equation (16), these hypotheses translate into the following parameter restrictions:

Hypothesis 4. The probability of a jump in stock returns is higher if there is little information about cash flows (few firms announce dividends) and if there was a large negative jump in the cash flow process. 


$$
H_{4}: \lambda_{2}^{r}<0 \text { and } \lambda_{3}^{r}<0
$$

\subsection{Empirical results}

Before testing the hypotheses laid out above, we briefly present results from estimating our model for dynamics in daily stock returns. ${ }^{24}$ To this end, Figure 8 shows the time series of $h_{r t+1}, J_{r t+1}$ and $\xi_{r t+1}$, extracted from daily stock returns using the model in equations (13)-(16). The top panel in Figure 8 shows that the volatility of stock returns rose markedly during the 2008-09 financial crisis as we would expect. The third and fourth panels indicate that also the jump intensity was notably higher during the financial crisis of 2008-09.

Table 5 reports posterior means and credible sets for the parameters of the return model. Our model identifies highly persistent, mean reverting components in both the conditional mean and volatility of returns with mean estimates $\phi_{\mu r}=0.989$ and $\phi_{h r}=0.990$ so that $99 \%$ of the daily value of the persistent mean or log-volatility component carries over to the following day. The jump intensity parameters suggest an average jump probability of $7.2 \%$, corresponding to a jump in stock returns occurring every 14 days. These features of our model for stock returns are consistent with similar ones identified in earlier studies such as Eraker et al. (2003).

Turning to the tests of the economic hypotheses listed in the previous subsection, consistent with $H_{1}$ the estimate of $\beta_{1}$ is highly statistically significant with the expected positive value, so that positive news about the persistent growth rate in cash flows are associated with higher stock returns on the same day. The coefficient on the jump component, $\beta_{3}$, is positive and significant at the $90 \%$ confidence level though it is insignificant at the $95 \%$ level. Thus, there is some evidence to suggest that jumps in the dividend growth process affect stock returns, contrary to $H_{1} \cdot{ }^{25}$ Finally, the insignificant and small value of $\beta_{4}$ suggests that temporary shocks to dividend growth do not directly affect same-day returns, consistent with $H_{1}$.

Our second hypothesis is that higher uncertainty about cash flow growth translates into

\footnotetext{
${ }^{24}$ As with the econometric model of Section 3, we provide the full details of our estimation procedure and converge statistics in Appendix A and Appendix B.

${ }^{25} \mathrm{~A}$ possible explanation of this finding is that the risk premium on stocks rises on days with negative jumps in the dividend growth process, leading to a downward adjustment in the stock price on such days.
} 
lower stock returns. Consistent with this hypothesis, our estimate of $\beta_{2}$ is positive and statistically significant so that we tend to find higher average returns on days with higher cash flow volatility. Importantly, this result is driven by an expected volatility effect: if we split the actual volatility $\exp \left(h_{d t+1} / 2\right)$ into an expected term, given information on day $t$, and the surprise component (i.e., the unanticipated change in volatility on day $t+1$ ), we find that only the expected cash flow volatility $\exp \left(h_{d t+1 \mid t} / 2\right)$ has a significant correlation with returns on day $t+1$.

Turning to the third hypothesis, consistent with $H_{3}$ we find that positive news about the persistent cash flow growth component tend to dampen return volatility $\left(\gamma_{1}<0\right.$ in (15)). Conversely, higher cash flow volatility or a negative jump in the cash flow growth rate are associated with significantly higher return volatility as $\gamma_{2}>0$ and $\gamma_{3}<0$.

Finally, our probit estimates of the probability of jumps in stock market returns (16) show that, consistent with $H_{4}$, the probability of observing a jump in returns is higher on days where few firms announce dividends ( $\lambda_{2}^{r}$ is negative) and on days with a negative jump in dividends (negative $\lambda_{3}^{r}$ ). Hence, a negative jump in the dividend growth process increases the probability of observing a jump in stock returns on the same day.

To evaluate the economic magnitude of how variation in $N_{d t+1}$ and $\xi_{d t+1} J_{d t+1}$ affect the probability of a jump in stock returns, we evaluate the fitted Probit model (16) at different percentiles of these variables. Setting the variables at their 25 th percentiles, we find a jump probability of $9 \%$, corresponding to a jump every 11 days. Conversely, setting these variables at their 75 th percentiles, the jump probability is $5.7 \%$. Hence, the chance of observing a jump in stock returns on a given day is notably higher if fewer firms announce earnings and if a negative jump affected the dividend growth process on that day.

We conclude from this evidence that the three different components of the cash flow growth process have distinctly different effects on the dynamics in the mean, volatility and jump probability of stock returns. News about the persistent component of dividend growth is associated with higher mean returns, whereas news about the temporary components has a much smaller effect on returns. Moreover, there is strong evidence that uncertainty surrounding the dividend growth process has an impact on the corresponding uncertainty measures of stock returns as the volatility and jump probability of the dividend growth 
process are significant drivers of the volatility and jump processes of returns.

\subsection{Uncertainty about dividend growth and expected returns}

Our second hypothesis suggests that uncertainty about cash flow growth should translate into lower stock prices through a higher risk premium. However, uncertainty about cash flow growth could also have a more direct effect on the uncertainty about expected returns. In particular, we would expect uncertainty about the persistent component of the mean dividend growth, $\mu_{d t}$, to be linked to uncertainty about the expected return (equity premium) process, $\mu_{r t}$.

Our approach allows us to readily compute an estimate of the uncertainty surrounding the two persistent components and we plot these estimates in Figure 9. Uncertainty about $\mu_{d t}$ rose sharply during the fall of 2008, remained at elevated levels until the third quarter of 2009, before declining during the last part of 2009 and early 2010. Uncertainty about $\mu_{r t}$ peaks in the third quarter of 2008 and comes down earlier than the corresponding uncertainty measure for $\mu_{d t}$.

\section{Robustness analysis}

This section conducts a set of tests designed to verify the robustness of the previous analysis. First, we report the outcome of simple regressions of daily stock returns on different measures of cash flow news. Moreover, we use these regressions to study the relation between stock returns and dividends on dividend payment days, rather than on days where dividends get announced. It is reasonable to expect the impact of dividends on stock returns to be largely associated with a news effect rather than a liquidity effect, which suggests that the effect on stock returns should be notably larger on announcement days than on payment days. Second, we estimate regressions of different measures of stock market volatility on the persistent cash flow component extracted from our dividend growth decomposition.

\subsection{Stock returns and cash flow news}

Our return model in equations (13)-(16) provides a structured framework for analyzing the relation between cash flow news and stock market returns. However, any inference will be 
model-dependent and so it is useful to explore whether our findings stand up in the context of a set of simple return regressions. To this end, we explore the robustness of the relation between stock returns and dividend growth news.

Because dividend growth news announced on day $t$ could arrive after markets have closed on that day, such news can affect aggregate stock market returns on day $t$ or day $t+1$ and so we measure trading day returns as the sum of the close-on-close returns on days $t$ and $t+1$, denoted $r_{t: t+1}$. To understand which, if any, of a set of alternative dividend growth measures are correlated with stock returns, we consider three different specifications. The first specification simply regresses two-day returns on dividend growth news on day $t, \Delta d_{t}$ :

$$
r_{t: t+1}=\alpha+\beta_{1} \Delta d_{t}+\varepsilon_{t: t+1}
$$

This regression uses a very noisy measure of cash flow news, mixing up temporary and persistent components in the dividend growth process which we would expect to have very different impact on returns. To separate these components, our second return regression uses changes in the persistent component from the no-jump model, $\Delta \mu_{d t}^{N J}$, in equation (7) as the regressor:

$$
r_{t: t+1}=\alpha+\beta_{1} \Delta \mu_{d t}^{N J}+\varepsilon_{t: t+1}
$$

We would expect to find a positive estimate of $\beta_{1}$ in (18). However, the estimate of $\mu_{d t}^{N J}$ extracted from the no-jump model remains very noisy which might confound the regression. To address this issue, our final model regresses returns on the different dividend growth components extracted from the general model:

$$
r_{t: t+1}=\alpha+\beta_{1} \Delta \mu_{d t}+\beta_{2} \xi_{d t} J_{d t}+\beta_{4} \varepsilon_{d t}+\varepsilon_{t: t+1} .
$$

Results from the return regressions in (17)-(19) are presented in Table 6 for both the short sample, 1973-2016 (columns 1-3) and for the long sample, 1927-2016 (columns 4-6.) We focus our discussion on the shorter sample but note that the results are very similar across the two sample periods.

The regression of daily stock market returns on the daily change in the dividend growth rate, $\Delta d_{t}$, uncovers no evidence of a statistically significant positive relation between stock 
returns and cash flow news. This conclusion carries over to the regression of returns on the change in the persistent cash flow component extracted from the simple model that ignores jumps, $\Delta \mu_{d t}^{N J}$. In contrast, we find a positive and highly significant (t-statistic of 2.09) relation between stock returns and changes in the persistent cash flow component, $\Delta \mu_{d t}$, extracted from the general model that allows for jumps. The $R^{2}$ from this regression $(0.10 \%)$ is low, though not immodest in the context of a daily return regression.

For the longer sample dating back to 1927 (columns 4-6), we continue to find that changes in daily dividends as well as changes in the persistent dividend growth component extracted from a model with no jumps are insignificantly correlated with aggregate stock market returns. In contrast, changes in our estimate of the persistent dividend growth component that accounts for jumps, $\Delta \mu_{t}$, are highly correlated with stock returns (t-statistic of 3.60 and $R^{2}=0.16 \%$ ).

Consistent with the empirical results in the previous section, we continue to find only weak evidence (one instance with significance at the $10 \%$ level) that the jump or diffusion components are significantly correlated with stock returns.

\subsubsection{Dividend Payments versus Announced Dividends}

Our results up to this point show that movements in aggregate stock returns and market volatility are related to dividend news on the date of the announcement. It is plausible that this relation reflects how investors re-assess equity prices following cash flow news. We can test this hypothesis by exploiting the fact that we have data on both the date of the dividend announcement and the date where a dividend is paid out, with the payment date typically occurring several days after the announcement date. If the news effect hypothesis is correct, we would expect to find a substantially smaller impact of dividend growth on stock returns on the payment date as compared to the return effect on the announcement date.

To see if this is the case, we estimate daily return regressions of the form in (19), but using the dividend payment date as opposed to the dividend announcement date. The results, presented in columns 7-9 of Table 6, show that the t-statistic on $\mu_{d t}$ drops from 3.60 to 2.01 and the $R^{2}$ values declines from $0.16 \%$ to $0.06 \%$. This is consistent with the cash flow news effect being what matters to movements in aggregate stock market prices, rather than any 
liquidity effects associated with payment of dividends.

\subsubsection{Analyst surprises and stock returns}

Our approach produces forecasts of daily cash flow growth from past dividend announcements, but there are also alternative estimates of cash flows. In particular we can use data on analyst forecasts to construct estimates of dividend surprises, using the approach of Patton and Verardo (2012) and Doyle et al. (2006). To this end, we construct a sample which includes all announced DPS available from IBES from July 1984 to January 2016 with at least two dividend forecasts. ${ }^{26}$ To keep as many observations as possible and limit the impact of outliers, we winsorize the data at the $1 \%$ level. Overall, our sample consists of 39,918 dividend surprises. For each day, we construct our aggregate daily dividend surprise measure $S U D_{t}$ by value-weighting individual firms' dividend surprises, as defined by

$$
S U D_{t}^{i}=\frac{\text { Actual DP } S_{t}^{i}-\text { Medest DP } S_{t}^{i}}{P_{t-7}^{i}}
$$

Here Medest $D P S_{t}$ is the last available median analyst DPS forecast ahead of the dividend announcement date, and $P_{t-7}$ is the stock price seven days before the actual DPS announcement. Using the stock price seven days prior to the dividend announcement date avoids the confounding effect of the announcement surprise on the stock price (Patton and Verardo (2012)).

Next, we regress the two-day cumulative return, $r_{t: t+1}$ on the values of $S U D_{t}$ and $\Delta \mu_{d t}$ from the general model with jumps:

$$
r_{t: t+1}=\alpha+\beta_{1} S U D_{t}+\beta_{2} \Delta \mu_{d t}+\varepsilon_{t: t+1} .
$$

Results from this regression reveal that our $\mu_{d t}$ measure continues to be positively correlated with stock market returns after controlling for analysts' dividend expectations, although the coefficient on $\mu_{d t}$ is only marginally significant with a t-statistic of 1.93 . Conversely, the $S U D$ measure fails to explain same-day stock market returns as the coefficient on this variable

\footnotetext{
${ }^{26}$ The announcement date is the anndats_act variable in the "Announcement Date of the Actual" from the Detail Actuals File.
} 
obtains a t-statistic below one.

This finding is perhaps not surprising as earnings and dividend surprises mainly have a cross-sectional effect: a daily dividend surprise announced by an individual firm can affect the stock itself - as well as stock prices of other companies within the same industry or geographical areas - by a lead-lag relationship (e.g., Parsons et al. (2017)), but does not seem to drive the return of the overall aggregate stock market. Hence, movement in the aggregate stock market cannot be explained by the $S U D$ value of individual stocks.

\subsection{Stock market volatility and dividend news}

Our empirical analysis in Section 5 shows that dividend growth dynamics affect not only the mean of stock returns but also have an impact on the volatility and jump probability of the return process. In particular, positive news about the persistent dividend growth component reduces stock market volatility. Conversely, higher dividend growth volatility leads to greater volatility in stock prices.

To explore the robustness of this finding, we investigate the relation between daily stock market volatility and cash flow news using two different measures of market volatility. First, we use the VIX obtained from options prices. The widely-used VIX measure reflects market expectations of short-run (30-day) volatility in stock prices. Second, we use a realized variance (RV) measure of daily stock market volatility based on intra-day movements in the price on the S\&P500 index sampled every 5 minutes. ${ }^{27}$ Data on the VIX are available starting in 1990, while data on realized volatility begin in 2000 .

We first consider the contemporaneous relation between daily stock market volatility and news about the persistent dividend growth component. Panel A in Table 7 shows that there is a significant and negative correlation between movements in the persistent dividend growth component and stock market volatility measured either by the VIX or by the RV, consistent with the notion that positive news about long-run dividend growth reduces volatility in the stock market.

Next, we consider whether dividend growth news helps predict future stock market

\footnotetext{
${ }^{27}$ Our data come from the Oxford-Man Institute of Quantitative Finance, see http://realized.oxford-man. ox.ac.uk/data/download.
} 
volatility. In common with empirical studies such as Paye (2012), we use the level of volatility in our regressions, but account for the high persistence in this variable by including either a single lag of volatility or an average of lagged volatility as proposed in the cascade model of Corsi (2009). Specifically, we use the following two regression specifications for the volatility on day $t, V O L_{t}$ :

$$
\begin{aligned}
V o l_{t+1} & =\alpha+\beta_{1} V o l_{t}+\beta_{2} \mu_{d t}+\varepsilon_{t+1}, \\
V o l_{t+1} & =\alpha+\beta_{d} R V_{t}^{d}+\beta_{w} R V_{t}^{w}+\beta_{m} R V_{t}^{m}+\beta \mu_{d t}+\varepsilon_{t+1},
\end{aligned}
$$

where $R V^{d}, R V^{w}$ and $R V^{m}$ are the daily, weekly, and monthly volatility averages, respectively, as defined in Corsi (2009).

Panel B in Table 7 shows the results from these regressions using the VIX (left column) or the realized volatility (right column). Independently of whether we use the regression specification in (22) or in (23), we find strong evidence of persistence in the volatility process. The VIX measure is particularly persistent which can be explained by its use of a partially overlapping 30-day horizon.

Turning to the predictive content of the persistent dividend component, $\mu_{d t}$, over stock market volatility, for both specifications in Panel $\mathrm{B}$ we find that the coefficient on $\mu_{d t}$ is negative and highly statistically significant with t-statistics of -4.40 and -9.27 , respectively. While these t-statistics drop to -2.31 and -2.21 in the cascade model, they remain significant at the $5 \%$ level. This suggests that positive news about persistent dividend growth lead to lower stock market volatility, while negative news tend to increase stock market volatility. ${ }^{28}$

\section{Conclusion}

This paper develops a new methodology for constructing daily estimates of cash flow growth based on firms' announcements of dividends. Two stylized facts characterize the cash flows that get announced on a given day. First, the number of firms that pay dividends often

\footnotetext{
${ }^{28}$ We also analyze whether the stochastic volatility and jump components extracted from the jump model have any contemporaneous or predictive effect on the aggregate volatility but find that the effects are negligible and not statistically significant.
} 
changes substantially from day to day. Second, individual firms' cash flow news can change by large amounts from one quarter to the next and are highly heterogeneous across firms. Both effects cause lumpiness in the daily cash flow news process.

We handle this lumpiness by decomposing news on dividend growth into a transitory "normal" shock whose volatility can vary over time, jumps that occur more rarely but whose magnitude tends to be much larger, and a persistent, smoothly evolving component that captures long-run mean dynamics in the cash flow growth process. We find that these components are well identified in the dividend growth data. Importantly, the persistent mean component captures predictable dynamics in daily dividend growth which gets overlooked if one studies the raw dividend growth series because this is dominated by the more volatile temporary components. In turn, we show empirically that this persistent dividend growth component can be used to produce more accurate forecasts of future dividend growth than alternative approaches from the existing finance literature.

Our model for daily cash flow dynamics opens up for new tests of asset pricing models conducted at a higher frequency than conventional tests and our analysis take a number of steps in this direction. Specifically, we formulate a dynamic model that allows us to study the effect of different cash flow news components on the conditional distribution of stock market returns. We find that positive news about the persistent component of dividend growth is associated with significantly higher average stock returns, while news about the temporary components has a much smaller effect on returns. Higher cash flow volatility and negative jumps in the cash flow process are also associated with higher volatility in the stock market and a higher probability of observing a jump in returns. 


\section{References}

Albert, James H., and Siddhartha Chib, 1993, Bayesian analysis of binary and polychotomous response data, Journal of the American Statistical Association 88, 669-679.

Andersen, Torben G., Tim Bollerslev, Francis X. Diebold, and Clara Vega, 2007, Real-time price discovery in global stock, bond and foreign exchange markets, Journal of International Economics 73(2), 251-277.

Aruoba, S. Boragan, Francis X. Diebold, and Chiara Scotti, 2009, Real-time measurement of business conditions, Journal of Business and Economic Statistics 27(4), 417-427.

Baker, Scott R., Nicholas Bloom, and Steven J. Davis, 2016, Measuring economic policy uncertainty, The Quarterly Journal of Economics .

Bansal, Ravi, and Amir Yaron, 2004, Risks for the long run: A potential resolution of asset pricing puzzles, The Journal of Finance 59(4), 1481-1509.

Becker, Bo, and Victoria Ivashina, 2014, Cyclicality of credit supply: Firm level evidence, Journal of Monetary Economics 62, 76-93.

Boyd, John H., Jian Hu, and Ravi Jagannathan, 2005, The stock market's reaction to unemployment news: Why bad news is usually good for stocks, The Journal of Finance 60(2), 649-672.

Campbell, JY, and RJ Shiller, 1988, The dividend-price ratio and expectations of future dividends and discount factors, Review of Financial Studies 1, 195-228.

Cavallo, Alberto, and Roberto Rigobon, 2016, The billion prices project: Using online data for measurement and research, Journal of Economic Perspectives 31(1), 151-178.

Chan, Joshua C. C., and Angelia L. Grant, 2016a, On the observed-data deviance information criterion for volatility modeling, Journal of Financial Econometrics 14, 772.

Chan, Joshua C.C., and Angelia L. Grant, 2016b, Modeling energy price dynamics: GARCH versus stochastic volatility, Energy Economics 54, 182 - 189. 
Chib, Siddhartha, Federico Nardari, and Neil Shephard, 2002, Markov chain monte carlo methods for stochastic volatility models, Journal of Econometrics 108, $281-316$.

Cochrane, John H., 1992, Explaining the variance of price-dividend ratios, The Review of Financial Studies 5(2), 243-280.

Cochrane, John H., 2008, The dog that did not bark: a defense of return predictability, The

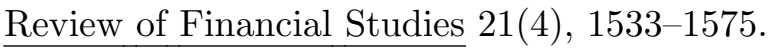

Cochrane, John H., 2011, Presidential address: Discount rates, The Journal of Finance 66(4), $1047-1108$.

Corsi, Fulvio, 2009, A simple approximate long-memory model of realized volatility, Journal of Financial Econometrics 7(2), 174-196.

Cutler, David M., James M. Poterba, and Lawrence H. Summers, 1989, What moves stock prices?, The Journal of Portfolio Management 15(3), 4-12.

Doyle, Jeffrey T., Russell J. Lundholm, and Mark T. Soliman, 2006, The extreme future stock returns following i/b/e/s earnings surprises, Journal of Accounting Research 44(5), $849-887$.

Eisfeldt, Andrea, and Tyler Muir, 2016, Aggregate external financing and savings waves, Working Paper .

Eraker, Bjrn, Michael Johannes, and Nicholas Polson, 2003, The impact of jumps in volatility and returns, The Journal of Finance 58, 1269-1300.

Gilchrist, Simon, and Egon Zakrajek, 2012, Credit spreads and business cycle fluctuations, American Economic Review 102(4), 1692-1720.

Hong, Harrison, and Jeffrey D. Kubik, 2003, Analyzing the analysts: Career concerns and biased earnings forecasts, The Journal of Finance 58(1), 313-351.

Hu, Grace Xing, Jun Pan, and Jiang Wang, 2013, Noise as information for illiquidity, The Journal of Finance 68(6), 2341-2382. 
Jurado, Kyle, Sydney C. Ludvigson, and Serena Ng, 2015, Measuring uncertainty, American Economic Review 105(3), 1177-1216.

Kelly, Bryan, and Seth Pruitt, 2013, Market expectations in the cross-section of present values, The Journal of Finance 68(5), 1721-1756.

Kim, Sangjoon, Neil Shephard, and Siddhartha Chib, 1998, Stochastic volatility: Likelihood inference and comparison with ARCH models, The Review of Economic Studies 65, 361393.

Koijen, Ralph S.J., and Stijn Van Nieuwerburgh, 2011, Predictability of returns and cash flows, Annual Review of Financial Economics 3, 467-491.

Kragt, Jac, Frank De Jong, and Joost Driessen, 2015, The dividend term structure, Working Paper .

Lettau, Martin, and Sydney Ludvigson, 2005, Expected returns and expected dividend growth, Journal of Financial Economics 76(3), 583-626.

Lettau, Martin, and Stijn Van Nieuwerburgh, 2008, Reconciling the return predictability evidence, The Review of Financial Studies 21(4), 1607-1652.

Liew, Jimmy, and Maria Vassalou, 2000, Can book-to-market, size and momentum be risk factors that predict economic growth?, Journal of Financial Economics 57, 221-245.

Lim, Terence, 2001, Rationality and analysts' forecast bias, The Journal of Finance 56(1), $369-385$.

Maio, Paulo, and Pedro Santa-Clara, 2015, Dividend yields, dividend growth, and return predictability in the cross-section of stocks, Journal of Financial and Quantitative Analysis $50(1-2), 33-60$.

McQueen, Grant, and V. Vance Roley, 1993, Stock prices, news, and business conditions,

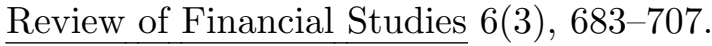

Parsons, Christopher A., Riccardo Sabbatucci, and Sheridan Titman, 2017, Geographic momentum, Working Paper . 
Patton, Andrew J., and Michela Verardo, 2012, Does beta move with news? firm-specific information flows and learning about profitability, The Review of Financial Studies 25(9), $2789-2839$.

Paye, Bradley S., 2012, "déjà vol:" predictive regressions for aggregate stock market volatility using macroeconomic variables, Journal of Financial Economics 106(3), 527-546.

Sabbatucci, Riccardo, 2017, Are dividends and stock returns predictable? new evidence using m\&a cash flows, Working Paper .

Savor, Pavel, and Mungo Wilson, 2016, Earnings announcements and systematic risk, The Journal of Finance 71(1), 83-138.

van Binsbergen, Jules H., Michael Brandt, and Ralph S. J. Koijen, 2012, On the timing and pricing of dividends, American Economic Review 102(4), 1596-1618.

van Binsbergen, Jules H., and Ralph S. J. Koijen, 2010, Predictive regressions: A presentvalue approach, The Journal of Finance 65(4), 1439-1471.

van Binsbergen, Jules H., and Ralph S. J. Koijen, 2016, The term structure of returns: Facts and theory, Journal of Financial Economics .

Working, Holbrook, 1960, Note on the correlation of first differences of averages in a random chain, Econometrica 28(4), 916-918. 


\begin{tabular}{|c|c|c|c|c|c|c|}
\hline \multicolumn{7}{|c|}{ Parameter estimates } \\
\hline & \multicolumn{3}{|c|}{ 1973-2016 } & \multicolumn{3}{|c|}{ 1927-2016 } \\
\hline & Mean & $S t d$ & 90\% Credible Set & Mean & $S t d$ & 90\% Credible Set \\
\hline$\mu_{d}$ & 0.084 & 0.015 & {$[0.064,0.104]$} & 0.062 & 0.016 & {$[0.036,0.088]$} \\
\hline$\phi_{\mu}$ & 0.998 & 0.001 & {$[0.997,0.999]$} & 0.998 & 0.000 & {$[0.998,0.999]$} \\
\hline$\sigma_{\mu}$ & 0.002 & 0.000 & {$[0.002,0.003]$} & 0.003 & 0.000 & {$[0.003,0.004]$} \\
\hline$\mu_{h}$ & -3.385 & 0.052 & {$[-3.470,-3.299]$} & -3.106 & 0.041 & {$[-3.174,-3.038]$} \\
\hline$\phi_{h}$ & 0.963 & 0.002 & {$[0.959,0.967]$} & 0.967 & 0.002 & {$[0.964,0.969]$} \\
\hline$\sigma_{h}$ & 0.205 & 0.001 & {$[0.203,0.207]$} & 0.203 & 0.001 & {$[0.202,0.205]$} \\
\hline$\sigma_{\xi}$ & 1.437 & 0.165 & {$[1.191,1.732]$} & 1.149 & 0.084 & {$[1.017,1.290]$} \\
\hline$\lambda_{1}$ & -1.589 & 0.056 & {$[-1.684,-1.497]$} & -1.576 & 0.036 & {$[-1.635,-1.516]$} \\
\hline$\lambda_{2}$ & -0.025 & 0.003 & {$[-0.030,-0.021]$} & -0.033 & 0.003 & {$[-0.038,-0.029]$} \\
\hline
\end{tabular}

Table 1: Parameter estimates for the dividend growth rate model. This table shows parameter estimates for a dynamic model fitted to the daily series of year-on-year dividend growth. The equations for the components model, further described in Section 3.2, take the following form:

$$
\begin{gathered}
\Delta d_{t+1}=\mu_{d t+1}+\xi_{d t+1} J_{d t+1}+\varepsilon_{d t+1} \\
\mu_{d t+1}=\mu_{d}+\phi_{\mu}\left(\mu_{d t}-\mu_{d}\right)+\sigma_{\mu} \varepsilon_{\mu t+1} \\
\varepsilon_{d t+1} \sim \mathcal{N}\left(0, e^{h_{d t+1}}\right) \\
h_{d t+1}=\mu_{h}+\phi_{h}\left(h_{d t}-\mu_{h}\right)+\sigma_{h} \varepsilon_{h t+1} \\
\operatorname{Pr}\left(J_{d t+1}=1\right)=\Phi\left(\lambda_{1}+\lambda_{2} N_{d t+1}\right) \\
\xi_{d t+1} \sim \mathcal{N}\left(0, \sigma_{\xi}^{2}\right)
\end{gathered}
$$

where $\mu_{d t+1}$ captures the mean of the smooth component of the underlying dividend process, $J_{d t+1} \in\{0,1\}$ is a jump indicator that equals unity in case of a jump in dividends and otherwise is zero, $\xi_{d t+1}$ measures the jump size, $\varepsilon_{d t+1}$ is a temporary cash flow shock, $\varepsilon_{\mu t+1} \sim \mathcal{N}(0,1)$ is assumed to be uncorrelated at all times with the innovation in the temporary dividend growth component, $\varepsilon_{d t+1}$ and $\left|\phi_{\mu}\right|<1$. $h_{d t+1}$ denotes the log-variance of $\varepsilon_{d t+1}$, and $\varepsilon_{h t+1} \sim \mathcal{N}(0,1)$ is uncorrelated at all times with both $\varepsilon_{d t+1}$ and $\varepsilon_{\mu t+1} . \quad N_{d t+1}$ denotes the number of firms announcing their dividends at time $t+1$, while $\Phi$ stands for the CDF of a standard Normal distribution. The magnitude of the jumps is modeled as $\xi_{d t+1} \sim \mathcal{N}\left(0, \sigma_{\xi}^{2}\right)$. The columns report the posterior mean, standard deviation and $90 \%$ credible sets of the parameter estimates. 


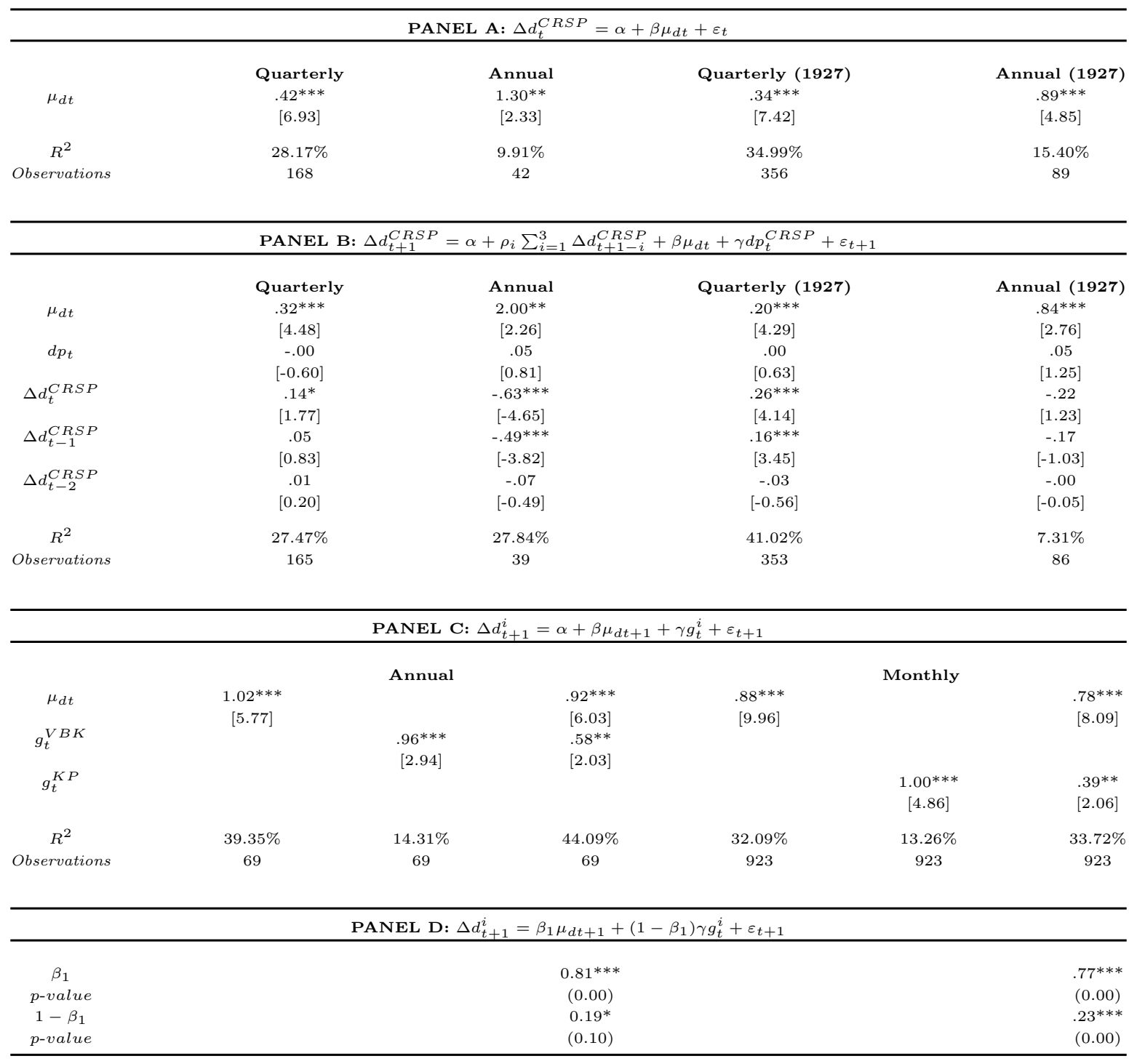

Table 2: Dividend growth regressions. Panel A reports results from contemporaneous regressions of the conventional CRSP dividend growth measure, $\Delta d_{t}^{C R S P}$, on the persistent dividend component $\mu_{d t}$ extracted from our daily dividend growth model. We report results for quarterly and annual frequencies, using the second half of our sample (1973-2016) as well as the full sample (1927-2016). Panel B reports results from predictive regression of $\Delta d_{t+1}^{C R S P}$ on the persistent component $\mu_{d t}$ estimated from our daily dividend growth model and the log dividend price ratio, $d p_{t}$, at quarterly and annual frequencies. Panel $\mathrm{C}$ compares the predictive power of our persistent dividend growth component to that of two latent dividend growth variables proposed in the literature. The first measure is taken from van Binsbergen and Koijen (2010) and uses cash reinvested dividend growth, measured annually over the extended sample period 1946-2015. The second measure is taken from Kelly and Pruitt (2013) and uses monthly data over the extended sample period 1940-2016. Panel D reports the results from forecast encompassing regressions which compares the predictive power of our $\mu_{d t}$ measure to the two alternative measures. Square brackets report t-statistics computed using Newey-West standard errors with three lags. 
PANEL A: $\Delta Y_{t}=\alpha+\beta \mu_{d t}+\varepsilon_{t}$

\begin{tabular}{|c|c|c|c|c|}
\hline \multirow{3}{*}{$\mu_{d t}$} & \multicolumn{2}{|c|}{$\Delta G D P$} & \multicolumn{2}{|c|}{$\Delta$ Consumption } \\
\hline & $.14^{* * *}$ & \multicolumn{3}{|c|}{$.13^{* * *}$} \\
\hline & {$[6.05]$} & \multicolumn{3}{|c|}{$[5.75]$} \\
\hline$R^{2}$ & $26.11 \%$ & \multicolumn{3}{|c|}{$30.50 \%$} \\
\hline Observations & 172 & \multicolumn{3}{|c|}{172} \\
\hline \multicolumn{5}{|c|}{ PANEL B: $\Delta Y_{t+1}=\alpha+\beta \mu_{d t}+\gamma \Delta Y_{t}+\varepsilon_{t+1}$} \\
\hline & \multicolumn{2}{|c|}{$\Delta G D P$} & \multicolumn{2}{|c|}{$\Delta$ Consumption } \\
\hline$\mu_{d t}$ & $.14^{* * *}$ & $.08^{* * *}$ & $.13^{* *}$ & $.03^{*}$ \\
\hline & {$[4.57]$} & {$[2.72]$} & {$[4.72]$} & {$[1.80]$} \\
\hline \multirow[t]{2}{*}{$\Delta Y_{t}$} & & $.39^{* * *}$ & & $.69^{* * *}$ \\
\hline & & {$[4.39]$} & & {$[11.77]$} \\
\hline$R^{2}$ & $21.15 \%$ & $32.82 \%$ & $25.93 \%$ & $59.53 \%$ \\
\hline Observations & 171 & 171 & 171 & 171 \\
\hline
\end{tabular}

Table 3: Contemporaneous and predictive regressions of GDP and consumption growth on the persistent dividend growth component. Panel A reports estimates from regressions of contemporaneous quarterly GDP and consumption growth, $\Delta Y_{t}$, on the persistent dividend growth component $\mu_{d t}$ estimated from our dynamic cash flow model. Panel B reports estimates from quarterly predictive regression of future GDP and consumption growth, $\Delta Y_{t}$, on the persistent dividend growth component $\mu_{d t}$ estimated from our dynamic cash flow model. Square brackets show t-statistics computed using Newey-West standard errors with three lags. The sample period used for these regressions is 1973-2015. 


\begin{tabular}{|c|c|c|c|c|c|c|}
\hline \multicolumn{7}{|c|}{ PANEL A: Index level correlations } \\
\hline & $V I X$ & $P U$ & $A D S$ & Liquidity & Inflation & $\mu_{d t}$ \\
\hline$V I X$ & 1 & & & & & \\
\hline$P U$ & $\begin{array}{c}0.34^{* * *} \\
(0.00)\end{array}$ & 1 & & & & \\
\hline$A D S$ & $\begin{array}{c}-0.49^{* * *} \\
(0.00)\end{array}$ & $\begin{array}{c}-0.26^{* * *} \\
(0.00)\end{array}$ & 1 & & & \\
\hline Liquidity & $\begin{array}{c}0.67^{* * *} \\
(0.00)\end{array}$ & $\begin{array}{c}0.19^{* * *} \\
(0.00)\end{array}$ & $\begin{array}{c}-0.54^{* * *} \\
(0.00)\end{array}$ & 1 & & \\
\hline Inflation & $\begin{array}{c}-0.66^{* * *} \\
(0.00)\end{array}$ & $\begin{array}{c}-0.36^{* * *} \\
(0.00)\end{array}$ & $\begin{array}{c}0.50^{* * *} \\
(0.00)\end{array}$ & $\begin{array}{c}-0.63^{* * *} \\
(0.00)\end{array}$ & 1 & \\
\hline$\mu_{d t}$ & $\begin{array}{c}-0.53^{* * *} \\
(0.00)\end{array}$ & $\begin{array}{c}-0.23^{* * *} \\
(0.00)\end{array}$ & $\begin{array}{c}0.32^{* * *} \\
(0.00)\end{array}$ & $\begin{array}{c}-0.59^{* * *} \\
(0.00)\end{array}$ & $\begin{array}{c}0.78^{* * *} \\
(0.00)\end{array}$ & 1 \\
\hline \multicolumn{7}{|c|}{ PANEL B: Index changes/returns correlations } \\
\hline$\Delta V I X$ & $\begin{array}{c}\Delta V I X \\
1\end{array}$ & $\triangle P U$ & $\triangle A D S$ Index & $\Delta$ Liquidity & $\Delta$ Inflation & $\Delta \mu_{d t}$ \\
\hline$\triangle P U$ & $\begin{array}{l}-0.02 \\
(0.10)\end{array}$ & 1 & & & & \\
\hline$\triangle A D S$ Index & $\begin{array}{c}0.01 \\
(0.65)\end{array}$ & $\begin{array}{c}0.00 \\
(0.94)\end{array}$ & 1 & & & \\
\hline$\Delta$ Liquidity & $\begin{array}{l}-0.01 \\
(0.53)\end{array}$ & $\begin{array}{c}0.05^{* * *} \\
(0.00)\end{array}$ & $\begin{array}{l}-0.00 \\
(0.88)\end{array}$ & 1 & & \\
\hline$\Delta$ Inflation & $\begin{array}{c}0.03 \\
(0.24)\end{array}$ & $\begin{array}{l}-0.03 \\
(0.27)\end{array}$ & $\begin{array}{l}-0.00 \\
(0.87)\end{array}$ & $\begin{array}{l}-0.00 \\
(0.91)\end{array}$ & 1 & \\
\hline$\Delta \mu_{d t}$ & $\begin{array}{l}-0.02 \\
(0.22)\end{array}$ & $\begin{array}{l}-0.01 \\
(0.58)\end{array}$ & $\begin{array}{c}0.05^{* * *} \\
(0.09)\end{array}$ & $\begin{array}{l}-0.01 \\
(0.25)\end{array}$ & $\begin{array}{c}0.09^{* * *} \\
(0.00)\end{array}$ & 1 \\
\hline
\end{tabular}

Table 4: Correlations between the persistent dividend growth component $\mu_{d t}$ and macroeconomic and financial activity measures. This table reports correlations between the persistent dividend growth component $\mu_{d t}$ extracted from our daily cash flow model and the following daily macroeconomic variables/indicators: the VIX index, the policy uncertainty index of Baker et al. (2016), the ADS index of Aruoba et al. (2009), the liquidity noise index of Hu et al. (2013), and the daily inflation index of Cavallo and Rigobon (2016). Daily data. Panel A correlates the levels of these variables, while Panel B correlates the change in the variables. 


\begin{tabular}{cccc} 
& Mean & Std & 90\% Credible Set \\
$\beta_{1}$ & 1.336 & 0.420 & {$[0.645,2.018]$} \\
$\beta_{2}$ & $0.144^{\dagger}$ & 0.080 & {$[0.016,0.271]$} \\
$\beta_{3}$ & $0.034^{\dagger}$ & 0.019 & {$[0.003,0.066]$} \\
$\beta_{4}$ & $-0.023^{\dagger}$ & 0.027 & {$[-0.068,0.021]$} \\
$\mu_{r}$ & $0.022^{\dagger}$ & 0.023 & {$[-0.015,0.061]$} \\
$\phi_{\mu r}$ & 0.989 & 0.001 & {$[0.987,0.990]$} \\
& & & \\
$\mu_{h r}$ & -9.554 & 0.056 & {$[-9.648,-9.462]$} \\
$\phi_{h r}$ & 0.990 & 0.000 & {$[0.990,0.990]$} \\
$\gamma_{1}$ & -17.916 & 1.286 & {$[-20.010,-15.768]$} \\
$\gamma_{2}$ & $0.050^{\dagger}$ & 0.013 & {$[0.029,0.072]$} \\
$\gamma_{3}$ & -0.109 & 0.011 & {$[-0.127,-0.091]$} \\
& & & \\
$\lambda_{1}^{r}$ & -1.206 & 0.076 & {$[-1.330,-1.084]$} \\
$\lambda_{2}^{r}$ & -0.010 & 0.002 & {$[-0.013,-0.007]$} \\
$\lambda_{3}^{r}$ & -0.611 & 0.298 & {$[-1.119,-0.139]$} \\
& & & {$[0.156,0.211]$} \\
$\sigma_{\mu}$ & 0.182 & 0.017 & {$[9.999,10.000]$} \\
$\sigma_{h}$ & 9.999 & 0.000 & {$[0.457,0.639]$} \\
$\sigma_{\xi_{r}}$ & 0.541 & 0.055 & \\
& & & \\
\hline & & &
\end{tabular}

Table 5: Parameter estimates for the dynamic return model that conditions on cash flow news. The table shows parameter estimates of the return model in Section 5.1 which relates dynamics in stock returns to the components extracted from our daily dividend growth model. The parameters listed in the table are taken from the following model specification:

$$
\begin{gathered}
r_{t+1}=\mu_{r t+1}+\xi_{r t+1} J_{r t+1}+\beta_{1} \Delta \mu_{d t+1}+\beta_{2} \exp \left(h_{d t+1} / 2\right)+\beta_{3}\left(\xi_{d t+1} J_{d t+1}\right)+\beta_{4} \varepsilon_{d t+1}+\varepsilon_{r t+1} \\
\mu_{r t+1}=\mu_{r}+\phi_{\mu r}\left(\mu_{r t}-\mu_{r}\right)+\sigma_{\mu r} \varepsilon_{r \mu t+1} \\
h_{r t+1}=\mu_{h r}+\phi_{h r}\left(h_{r t}-\mu_{h r}\right)+\gamma_{1} \Delta \mu_{d t+1}+\gamma_{2} h_{d t+1}+\gamma_{3}\left(\xi_{d t+1} J_{d t+1}\right)+\sigma_{h r} \varepsilon_{r h t+1} \\
\operatorname{Pr}\left(J_{r t+1}=1\right)=\Phi\left(\lambda_{1}^{r}+\lambda_{2}^{r} N_{d t+1}+\lambda_{3}^{r}\left(\xi_{d t+1} J_{d t+1}\right)\right) \\
\xi_{r t+1} \sim \mathcal{N}\left(0, \sigma_{\xi_{r}}^{2}\right)
\end{gathered}
$$

where $\mu_{r t+1}$ captures the persistent component in stock returns, $\xi_{r t+1} J_{r t+1}$ represent jumps in stock returns with $J_{r t+1} \in\{0,1\}$ being a jump indicator, while $\varepsilon_{r t+1} \sim \mathcal{N}\left(0, e^{h_{r t+1}}\right)$ is a diffusion term with time-varying $\log$ variance $h_{r t+1} . \beta_{1} \Delta \mu_{d t+1}$ captures the effect of persistent cash flow news on stock returns, while the three additional components, $\beta_{2} \exp \left(h_{d t+1} / 2\right)$, $\beta_{3}\left(\xi_{d t+1} J_{d t+1}\right)$ and $\beta_{4} \varepsilon_{d t+1}$ capture spillover effects on returns from the conditional volatility, jumps and idiosyncratic shock in the dividend growth process. $\varepsilon_{r \mu t+1} \sim \mathcal{N}(0,1)$ is assumed to be uncorrelated at all times with the innovation in the temporary return component, $\varepsilon_{r t+1}$ and $\left|\phi_{\mu r}\right|<1 . h_{d t+1}$ denotes the time-varying variance extracted from the dividend model, while $J_{d t+1}$ and $\xi_{d t+1}$ denote the time-varying jump probability and jump magnitude obtained from the dividend growth rate model. $\varepsilon_{r h t+1} \sim \mathcal{N}(0,1)$ is uncorrelated at all times with both $\varepsilon_{r t+1}$ and $\varepsilon_{r \mu t+1} \cdot N_{d t+1}$ is the number of firms announcing dividends. The columns report the posterior mean, standard deviation and $90 \%$ credible sets of the parameter estimates. The $\sigma$ estimates (estimates with the $\dagger$ symbol) have been multiplied by 1,000 (100) for better readability. The model is estimated using daily data over the sample period 1973-2016. 
Dividend announcement days

1973-2016

\begin{tabular}{cccc}
$\Delta d_{t}$ & .00 & & \\
$\Delta \mu_{d t}^{N J}$ & {$[0.40]$} & & \\
& & .00 & \\
$\Delta \mu_{d t}$ & & {$[0.40]$} & 2.31 \\
& & & {$[2.0$} \\
$\xi_{d t} J_{d t}$ & & & .00 \\
& & & {$[1.81]$} \\
$h_{d t} / 2$ & & & -.00 \\
& & & {$[-0.03]$} \\
$R^{2}$ & $0.00 \%$ & $0.00 \%$ & $0.10 \%$ \\
Observations & 9,037 & 9,037 & 9,037 \\
\hline
\end{tabular}

$1927-2016$

.00

$[0.22$

\begin{tabular}{l}
$2.31^{* *}$ \\
{$[2.09]$} \\
$.00^{*}$ \\
{$[1.81]$} \\
-.00 \\
{$[-0.03]$} \\
$0.10 \%$ \\
9,037 \\
\hline
\end{tabular}

Table 6: Daily regressions of stock returns on dividend news. This table reports estimates from regressions of daily stock market returns on 1) daily changes in our aggregate dividend growth measure $\left.\left(\Delta y_{t}\right) ; 2\right)$ the change in the persistent component $\Delta \mu_{d t}^{N J}$ extracted from a dividend growth model without jumps and stochastic volatility; or 3) the change in the persistent component $\Delta \mu_{d t}$ extracted from the dynamic dividend growth model that accounts for jumps and stochastic volatility. In each case, the dependent variable is the two-day cumulative log stock market return $r_{t: t+1}$ Columns 1-6 consider stock returns on the dates of the dividend news announcements, while columns 7-9 instead relate stock returns to dividend news on the days where the dividend payments are actually made. Columns 1-3 show results for the shorter 1973-2016 sample, while columns 4-9 show results for the longer sample, 1927-2016. Square brackets report t-statistics using Newey-West standard errors with three lags. 


\section{Panel A: Contemporaneous regressions}

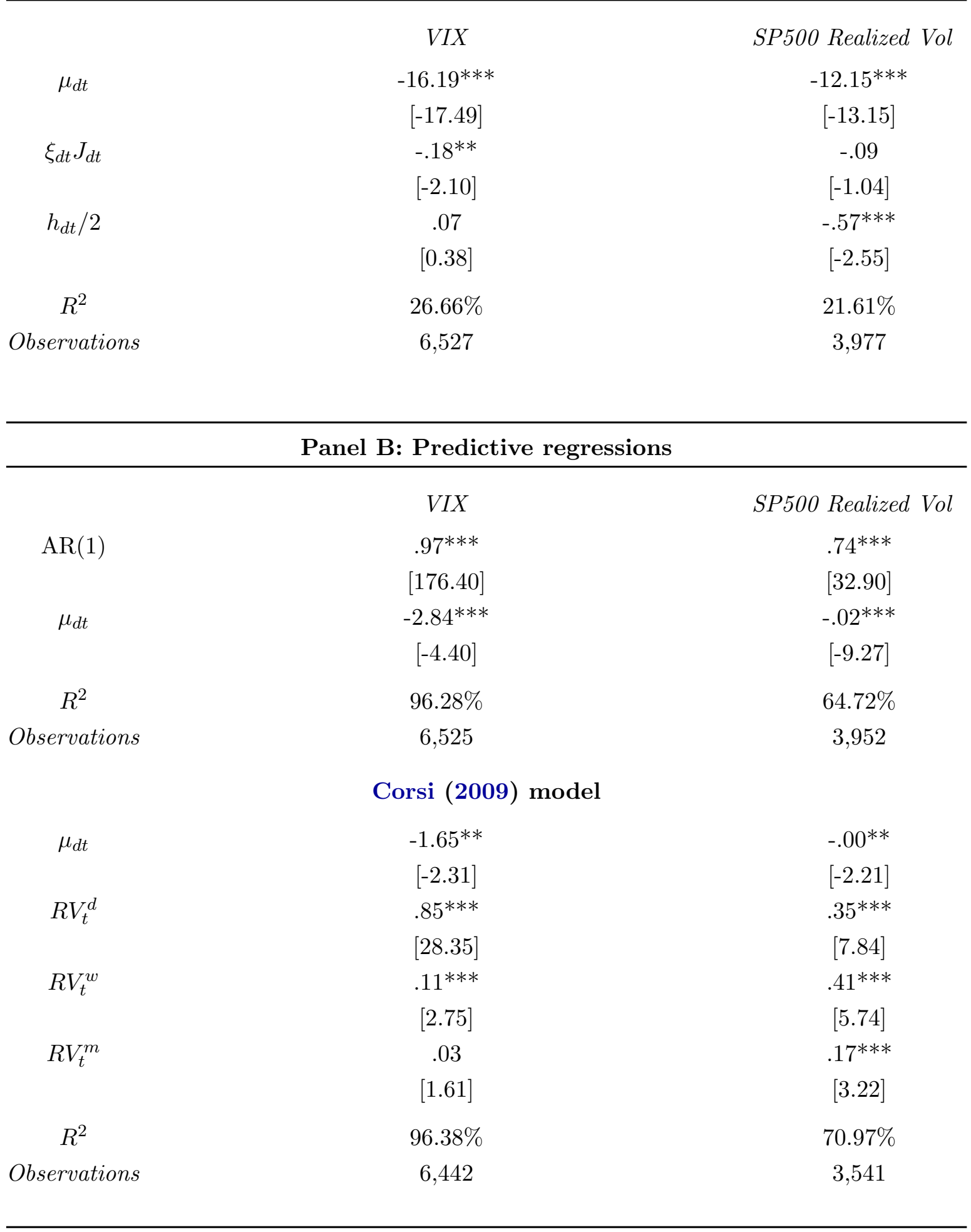

Table 7: Relation between the persistent dividend component, VIX, and realized stock market volatility. Panel A in this table reports estimates from daily regressions of the VIX (left column) or the realized volatility extracted from the S\&P500 index (right column) on the contemporaneous value of the persistent dividend growth component $\mu_{d t}$ extracted from our components model. Panel B reports similar results, relating the VIX or realized volatility to the lagged value of $\mu_{d t}$ as well as a single lag of the dependent variable or multiple lags based on the Corsi (2009) model. The dependent variables in Panel A are standardized. Square brackets show t-statistics using Newey-West standard errors computed using three lags. 

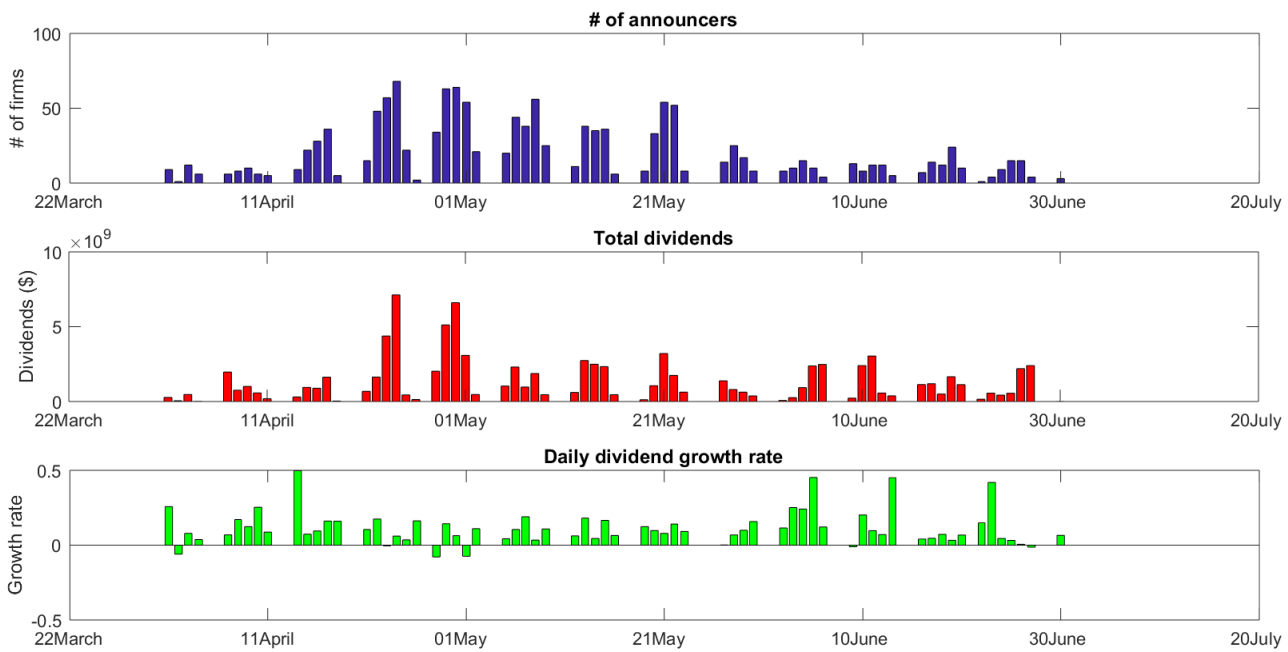

Figure 1: Distribution of dividend announcements within a quarter. This figure plots the time-series of dividend announcements for Q2 2014. For every day within this quarter, the top panel shows the number of firms announcing dividends. The middle panel shows the overall nominal amount of dividends announced by those firms, while the bottom panel shows the daily (net) dividend growth rate defined in equation (1). 

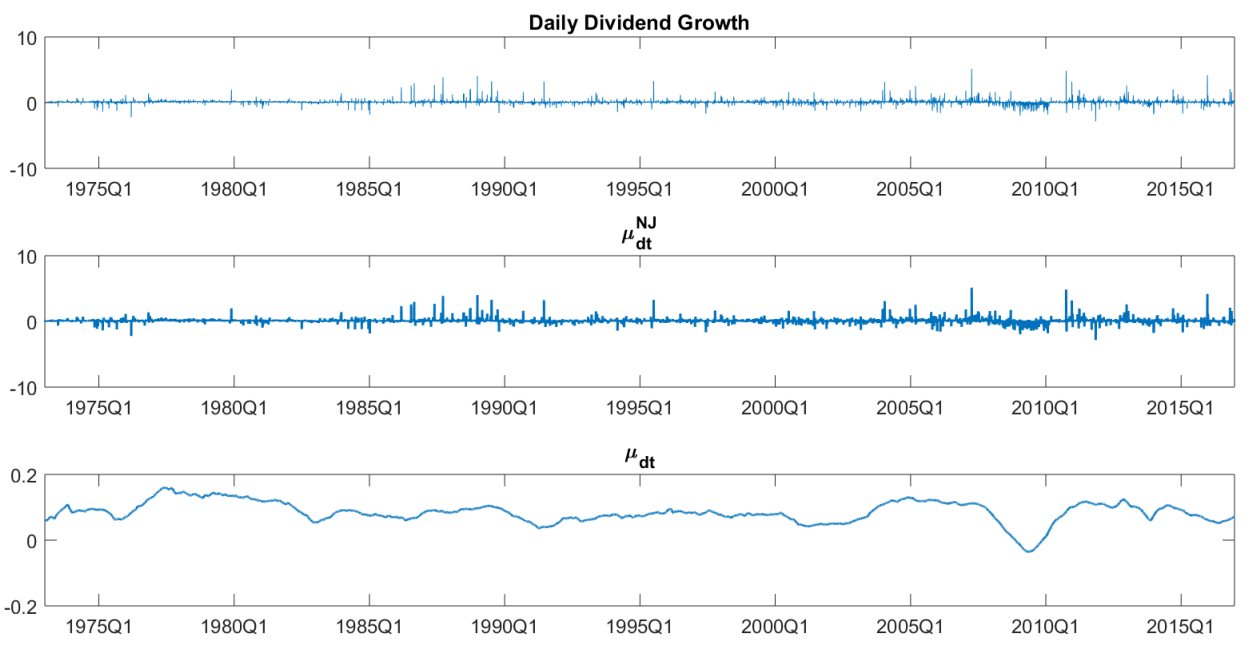

Figure 2: Time series of daily dividend growth and the persistent mean component. The top panel plots the log of the daily dividend growth series $D_{y r, s}$ defined in Eq.1. The middle panel plots the persistent dividend growth component $\mu_{d t}^{N J}$ extracted from a model without jumps and stochastic volatility. The bottom panel plots the persistent dividend growth component $\mu_{d t}$ extracted from the daily dividend series using a model that accounts for jumps and stochastic volatility. All plots use daily data over the sample 1973-2016.
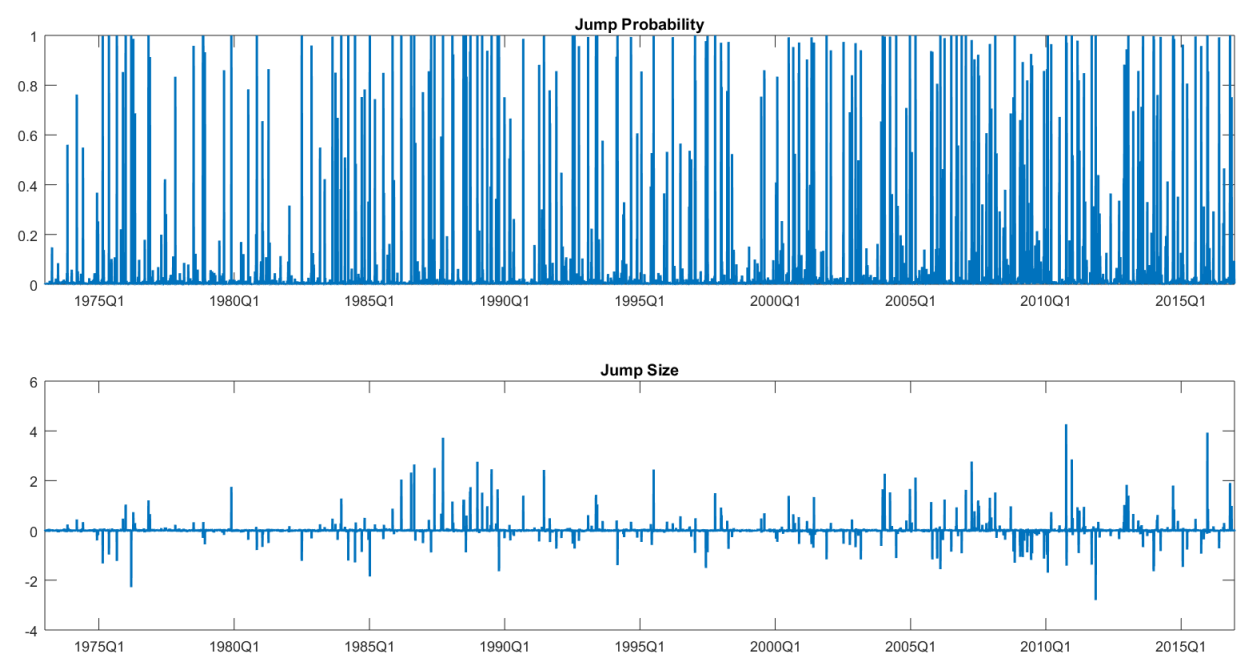

Figure 3: Jump dynamics in daily dividend news. The top panel plots the probability of a jump in the daily dividend growth series while the bottom panel plots the magnitude of such jumps. Both plots use daily dividend data over the sample 1973-2016. 


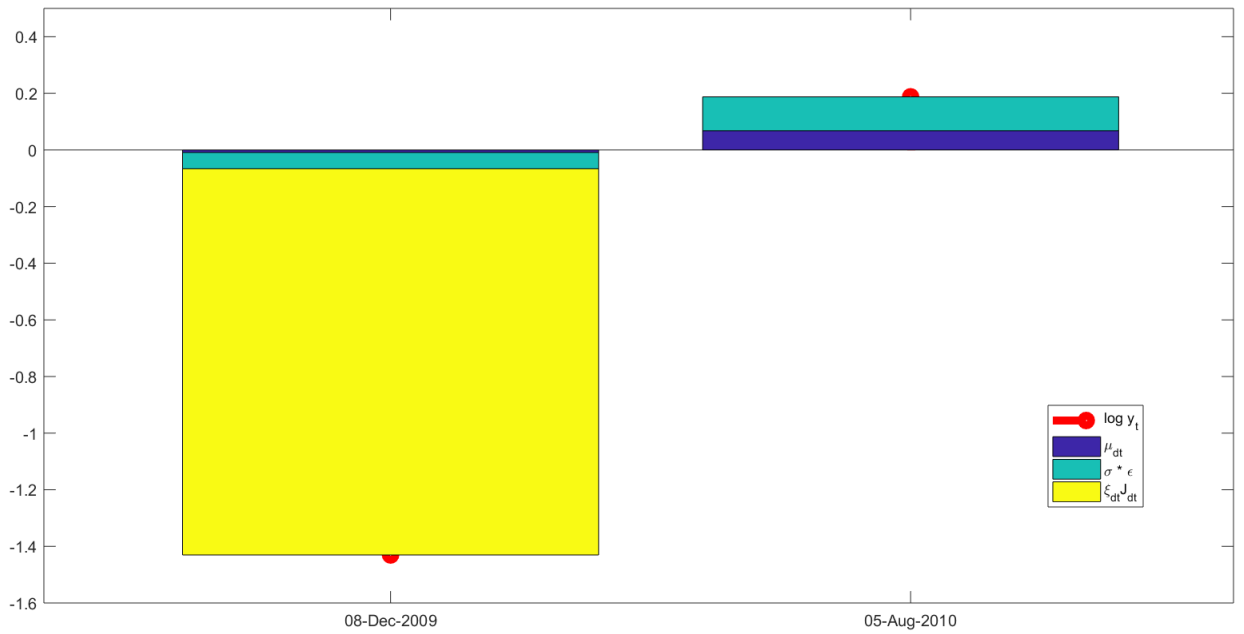

Figure 4: Decomposition of dividend growth into its components. For two days in our sample, this figure shows how the dividend growth rate gets decomposed into (i) a persistent mean component $\mu_{d t}$; (ii) a normal component with stochastic volatility $\sigma$; and (iii) a jump component. The two days are December 8, 2009 (a day during the global financial crisis) and August 5, 2010 (a day during the recovery). 


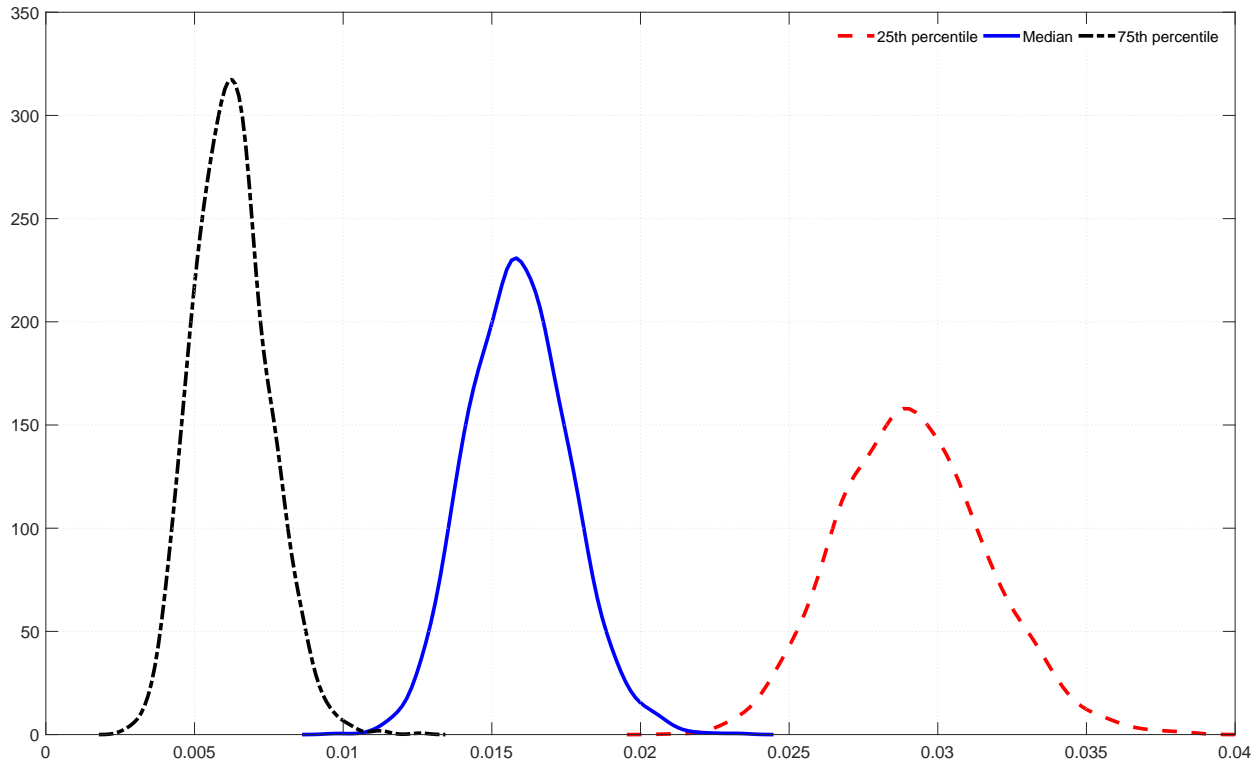

Figure 5: Jump intensities and the number of firms announcing dividend news. This figure shows the sensitivity of the dividend growth jump probability to the number of firms announcing dividends on a given day, $N_{d t}$, chosen to match the $25 \mathrm{th}$, median and 75 th percentiles of the distribution of the daily number of announcing firms. On days with a large number of announcing firms (black, dashed curve), the jump intensity distribution is centered around 0.005, corresponding to a jump on average every 200 days. On days with a typical (median) number of announcing firms (blue curve), the jump intensity is centered around 0.016, implying a jump roughly every 60 days. Finally, on days with a small number of announcing firms (red, dotted curve), the probability of a jump is 0.03 , corresponding to a jump every 35 days. 

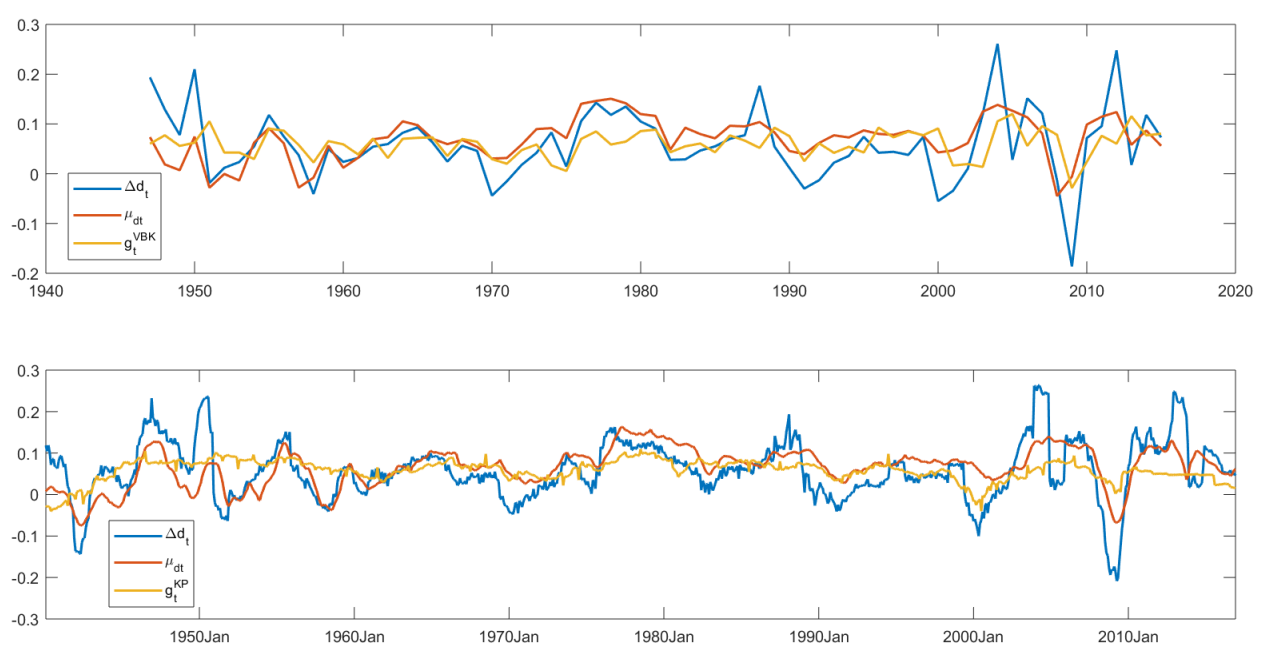

Figure 6: Actual versus predicted dividend growth under alternative modeling approaches. The top panel plots the actual dividend growth, $\Delta d_{t}$ against the persistent dividend growth component extracted from our model, $\mu_{d t}$ and the measure proposed by van Binsbergen and Koijen (2010), $g_{t}^{V B K}$. The latter assumes cash reinvested dividend growth. The bottom panel plots actual dividend growth against our persistent dividend growth component and the measure of Kelly and Pruitt (2013), $g_{t}^{K P}$. In both cases we have extended the sample period originally used by the papers after replicating their results.

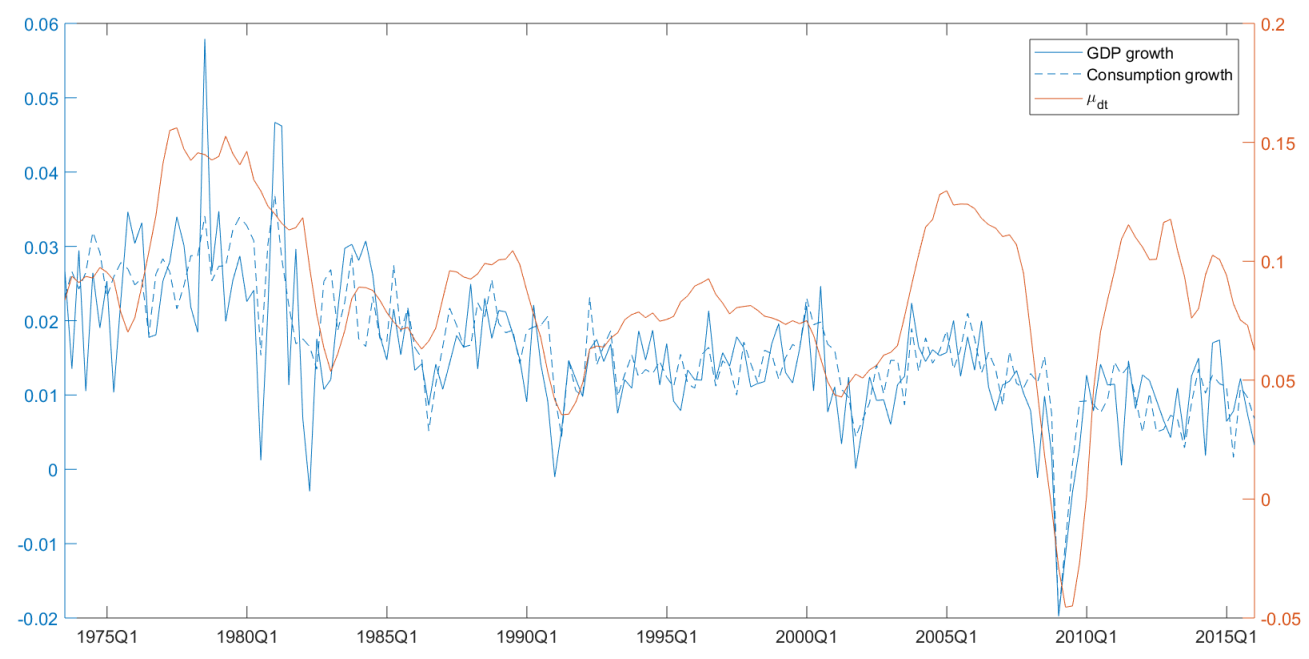

Figure 7: GDP growth, consumption growth and the persistent dividend growth component $\mu_{d t}$. This figure plots quarterly GDP and consumption growth along with the persistent dividend growth component $\mu_{d t}$, extracted from our daily cash flow model over the sample 1975-2015. 

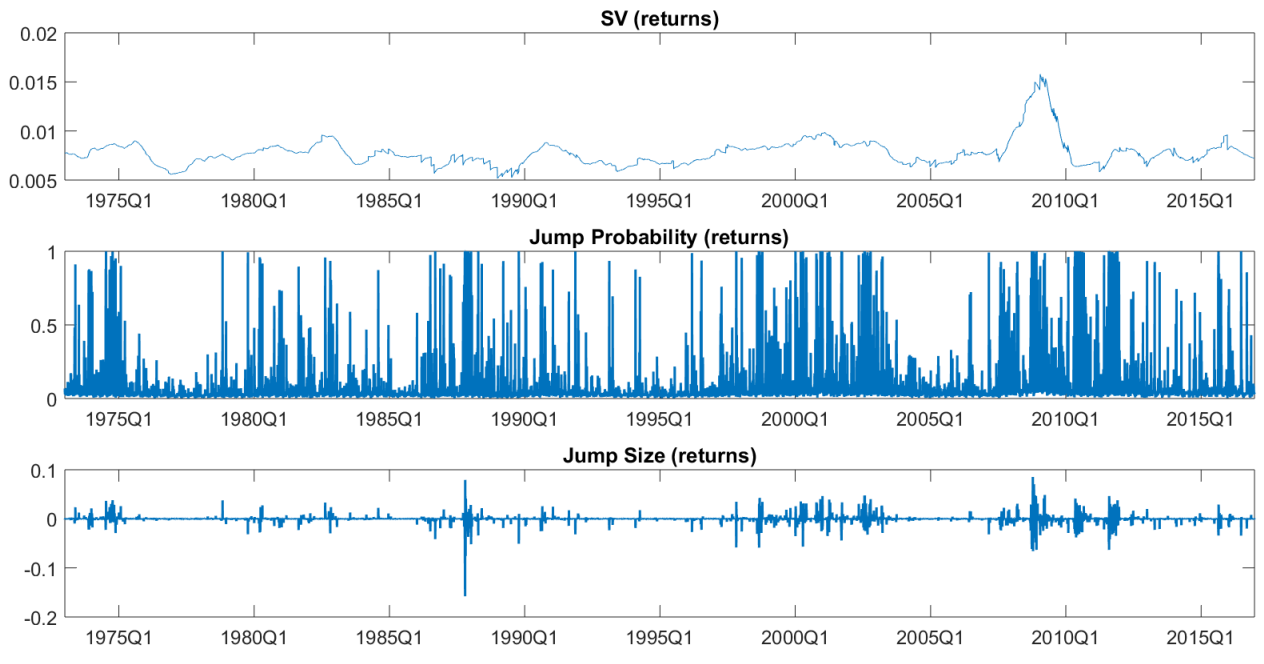

Figure 8: Decomposition of daily stock returns into stochastic volatility and jumps. The top panel of this figure shows the stochastic volatility (SV) component extracted from the dynamic model for stock returns (eq. 13). The middle and bottom panels plot the probability and magnitude of jumps in daily stock returns.
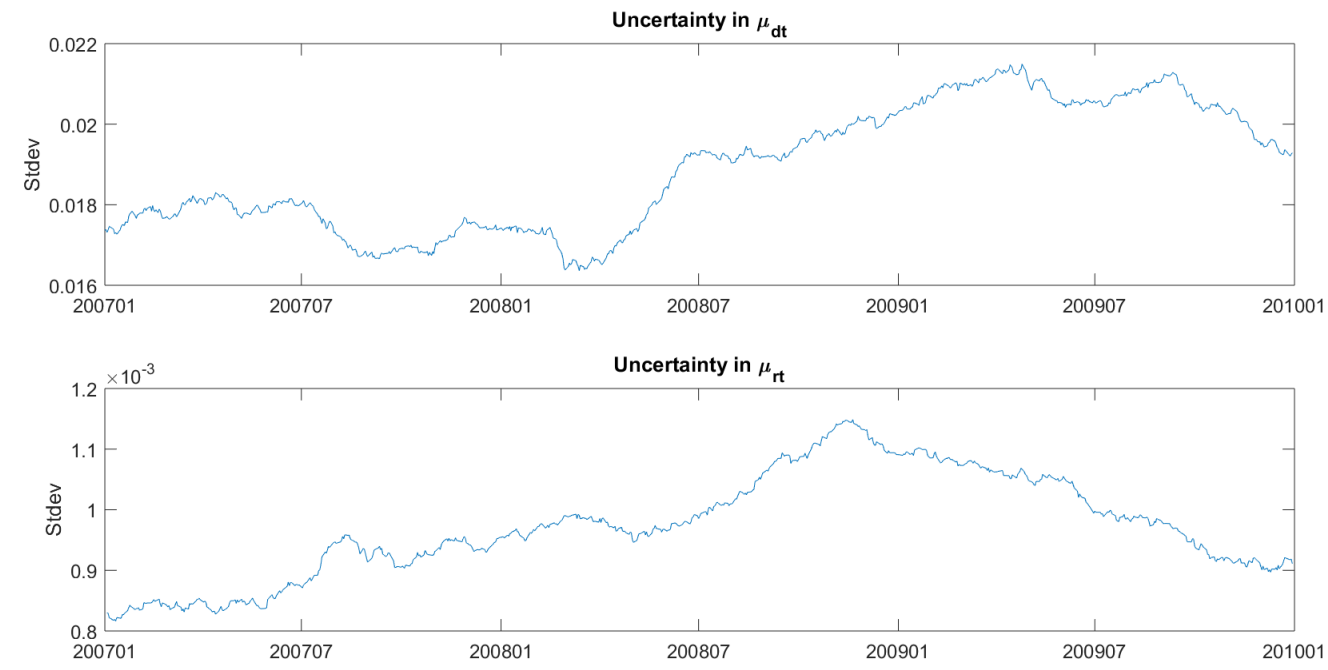

Figure 9: Uncertainty about the persistent mean components of dividend growth and returns. This graph plots the uncertainty surrounding the mean of the dividend growth process $\mu_{d t}$ and the mean of stock returns $\mu_{r t}$ during the period from 2007-2009. Uncertainty about dividend growth rises sharply during the fall of 2008, remains at elevated levels until the third quarter of 2009, before declining during the last part of 2009. Uncertainty is defined as the volatility of the daily estimates of $\mu_{d t}$ and $\mu_{r t}$, respectively. 


\section{Appendix A MCMC Algorithm}

In this Appendix, we provide the analytical derivations needed to derive the posterior distributions of all parameters and latent states of the most general model we estimate in the paper.

\section{A.1 The Model}

We start by rewriting both the model as well as the prior distributions for all model parameters. Starting with the observation equation and time-varying mean and volatility processes, we have

$$
\begin{gathered}
y_{t+1}=\mu_{y t+1}+\xi_{y t+1} J_{y t+1}+\boldsymbol{\beta}^{\prime} \boldsymbol{X}_{t+1}+\varepsilon_{y t+1}, \\
\mu_{y t+1}=\mu_{y}+\phi_{\mu}\left(\mu_{y t}-\mu_{y}\right)+\sigma_{\mu} \varepsilon_{\mu t+1},
\end{gathered}
$$

and

$$
h_{y t+1}=\mu_{h}+\phi_{h}\left(h_{y t}-\mu_{h}\right)+\boldsymbol{\beta}_{\boldsymbol{h}}{ }^{\boldsymbol{X}_{t+1}^{h}}+\sigma_{h} \varepsilon_{h t+1}
$$

where $y_{t+1}$ denotes either the cash flow growth rate at time $t+1\left(y_{t+1}=\Delta d_{t+1}\right)$ or the stock return at time $t+1\left(y_{t+1}=r_{t+1}\right)$, while $\varepsilon_{y t+1} \sim \mathcal{N}\left(0, e^{h_{y t+1}}\right), \varepsilon_{\mu t+1} \sim \mathcal{N}\left(0, \sigma_{\mu}^{2}\right)$, and $\varepsilon_{h t+1} \sim \mathcal{N}\left(0, \sigma_{h}^{2}\right)$ are mutually independent and independently and identically distributed across time. Next, the intensity of the jump process is assumed to take the form

$$
\operatorname{Pr}\left(J_{y t+1}=1\right)=\Phi\left(\boldsymbol{\lambda}^{\prime} \boldsymbol{X}_{t+1}^{J}\right),
$$

where $\Phi(\cdot)$ denotes the cumulative distribution function of a standard normal random variable, while the magnitude of the jumps is determined from

$$
\xi_{y t+1} \sim \mathcal{N}\left(0, \sigma_{\xi}^{2}\right)
$$


with $\boldsymbol{X}_{t+1}, \boldsymbol{X}_{t+1}^{h}$, and $\boldsymbol{X}_{t+1}^{j}$ exogenous. Finally, the initial conditions for $\mu_{y}$ and $h_{y}$ are set as follows:

$$
\mu_{y 1} \sim \mathcal{N}\left(\mu_{y}, \frac{\sigma_{\mu}^{2}}{1-\phi_{\mu}^{2}}\right)
$$

and

$$
h_{y 1} \sim \mathcal{N}\left(\mu_{h}+\frac{\boldsymbol{\beta}_{h}^{\prime} \boldsymbol{X}_{1}^{h}}{1-\phi_{h}}, \frac{\sigma_{h}^{2}}{1-\phi_{h}^{2}}\right) .
$$

\section{A.2 Priors}

The model in (A.1)-(A.7) includes 10 parameters, namely $\mu_{y}, \phi_{\mu}, \sigma_{\mu}^{2}, \mu_{h}, \phi_{h}, \sigma_{h}^{2}, \boldsymbol{\lambda}, \sigma_{\xi}^{2}, \boldsymbol{\beta}$, and $\boldsymbol{\beta}_{h}$. Accordingly, we specify the following prior distributions:

$$
\begin{gathered}
\mu_{y} \sim \mathcal{N}\left(\underline{\mu}_{y 0}, \underline{V}_{\mu_{y}}\right), \quad \phi_{\mu} \sim \mathcal{N}\left(\underline{\phi}_{\mu_{0}}, \underline{V}_{\phi_{\mu}}\right) I\left(\left|\phi_{\mu}\right|<1\right), \quad \sigma_{\mu}^{2} \sim \mathcal{I} \mathcal{G}\left(\underline{\nu}_{\mu}, \underline{S}_{\mu}\right) \\
\mu_{h} \sim \mathcal{N}\left(\underline{\mu}_{h 0}, \underline{V}_{\mu_{h}}\right), \quad \phi_{\mu} \sim \mathcal{N}\left(\underline{\phi}_{h_{0}}, \underline{V}_{\phi_{h}}\right) I\left(\left|\phi_{h}\right|<1\right), \quad \sigma_{h}^{2} \sim \mathcal{I} \mathcal{G}\left(\underline{\nu}_{h}, \underline{S}_{h}\right) \\
\boldsymbol{\lambda} \sim \mathcal{N}\left(\underline{\boldsymbol{\mu}}_{\boldsymbol{\lambda}}, \underline{\boldsymbol{V}}_{\boldsymbol{\lambda}}\right) \\
\sigma_{\xi}^{2} \sim \mathcal{I} \mathcal{G}\left(\underline{\nu}_{\xi}, \underline{S}_{\xi}\right) \\
\boldsymbol{\beta} \sim \mathcal{N}\left(\underline{\boldsymbol{\beta}}_{0}, \underline{\boldsymbol{V}}_{\boldsymbol{\beta}}\right) \\
\boldsymbol{\beta}_{h} \sim \mathcal{N}\left(\underline{\boldsymbol{\beta}}_{h 0}, \underline{\boldsymbol{V}}_{\boldsymbol{\beta}_{h}}\right) .
\end{gathered}
$$

where $\mathcal{N}$ is the normal distribution and $\mathcal{I} \mathcal{G}$ refers to the Inverse Gamma distribution.

Next, we briefly describe the choices of prior hyperparameters of the dividend growth and return models. Starting with the dividend growth model, for almost all of the parameters 
we work with loose and mildly uninformative priors. ${ }^{29}$ Starting with $\mu_{y}$ and $\mu_{h}$, we set $\underline{\mu}_{y_{0}}=\underline{\mu}_{h_{0}}=0$, and specify their variances as $\underline{V}_{\mu_{y}}=\underline{V}_{\mu_{h}}=10$. Next, we set $\underline{\phi}_{\mu_{0}}=\underline{\phi}_{h_{0}}=0.99$ and $\underline{V}_{\phi_{\mu}}=\underline{V}_{\phi_{h}}=0.001$, which implies a prior belief that the latent processes for $\mu_{y t+1}$ and $h_{y t+1}$ will be very persistent. Finally, we set $\underline{S}_{\mu}=0.01$ and $=\underline{S}_{h}=0.1$, restricting the changes to the process for $\mu_{y t+1}$ and $h_{y t+1}$ to be 0.01 and 0.1 on average, and set $\underline{\nu}_{\mu}=2$ and $\underline{\nu}_{h}=1 e^{10}$. As for the parameters of the jump process, we specify a normal prior for the parameters governing the timing of the jumps, i.e. $\boldsymbol{\lambda}=\left(\lambda_{1}, \lambda_{2}\right) \sim \mathcal{N}\left(\boldsymbol{\mu}_{\boldsymbol{\lambda}}, \boldsymbol{V}_{\boldsymbol{\lambda}}\right)$, where $\boldsymbol{\mu}_{\boldsymbol{\lambda}}=(0,0)^{\prime}$ and $\boldsymbol{V}_{\boldsymbol{\lambda}}=\operatorname{diag}(10,10)$. As for the magnitude of the jumps, we tailor the hyperparameter $\underline{S}_{\xi}=2^{2}$ to the spread of the series being modeled and set $\underline{\nu}_{\xi}=2$ to impose the least informative proper prior on $\sigma_{\xi}^{2}$.

Turning to the tuning of the priors for the return model, we set $\underline{\mu}_{y 0}=\underline{\mu}_{h_{0}}=0$, and specify their variances as $\underline{V}_{\mu_{y}}=\underline{V}_{\mu_{h}}=10$. Next, we set $\underline{\phi}_{\mu_{0}}=\underline{\phi}_{h_{0}}=0.99, \underline{V}_{\phi_{\mu}}=0.001$, and $\underline{V}_{\phi_{h}}=0.0001$. This implies a prior belief that the latent processes for $\mu_{r t+1}$ and $h_{r t+1}$ are highly persistent. Finally, we set $\underline{S}_{\mu}=0.001$ and $\underline{S}_{h}=0.01$, reflecting a belief that changes to $\mu_{r t+1}$ and $h_{r t+1}$ are very small and we set $\underline{\nu}_{\mu}=2$ and $\underline{\nu}_{h}=1 e^{10}$. Moreover, as for the dividend process, we specify a normal prior for the parameters governing the timing of the jumps, i.e., $\boldsymbol{\lambda}=\left(\lambda_{1}^{r}, \lambda_{2}^{r}, \lambda_{3}^{r}\right)$ and set $\boldsymbol{\mu}_{\boldsymbol{\lambda}}=(0,0,0)^{\prime}$ and $\boldsymbol{V}_{\boldsymbol{\lambda}}=\operatorname{diag}(10,10,10)$. Finally, we set $\underline{S}_{\xi}=0.025^{2}$ and $\underline{\nu}_{\xi}=2$. As for the remaining parameter in the mean and volatility equation, we specify a loosely uninformative normal prior with mean zero and variance equal to 10 for all the elements in the vectors $\boldsymbol{\beta}$ and $\boldsymbol{\beta}_{h}$.

\section{A.3 Posteriors}

We now describe how to obtain posterior estimates for all model parameters $\left(\mu_{y}, \phi_{\mu}, \sigma_{\mu}^{2}, \mu_{h}\right.$, $\left.\phi_{h}, \sigma_{h}^{2}, \boldsymbol{\lambda}, \sigma_{\xi}^{2}, \boldsymbol{\beta}, \boldsymbol{\beta}_{h}\right)$, as well as latent state vectors $\boldsymbol{\mu}_{y}=\left\{\mu_{y t}\right\}_{t=1}^{T}, \boldsymbol{h}_{y}=\left\{h_{y t}\right\}_{t=1}^{T}$, $\boldsymbol{J}_{y}=\left\{J_{y t}\right\}_{t=1}^{T}$, and $\boldsymbol{\xi}_{y}=\left\{\xi_{y t}\right\}_{t=1}^{T}$. While the joint posterior distribution of all model parameters and latent state variables is highly non-linear, we can employ a Gibbs sampler algorithm augmented with a number of Metropolis-Hastings steps to draw recursively from the conditional posteriors of all model parameter and state variables. In particular, we break the evaluation of the joint posterior distribution into five different blocks:

\footnotetext{
${ }^{29}$ Note that we impose the stationarity conditions $\left|\phi_{\mu}\right|<1$ and $\left|\phi_{h}\right|<1$ directly on the priors.
} 
1. $\boldsymbol{\mu}_{y} \mid \boldsymbol{h}_{y}, \boldsymbol{\xi}_{y}, \boldsymbol{J}_{y}, \mu_{y}, \phi_{\mu}, \sigma_{\mu}^{2}, \boldsymbol{\beta}, \mathcal{D}^{T}$

2. $\boldsymbol{J}_{y} \mid \boldsymbol{\mu}_{y}, \boldsymbol{\xi}_{y}, \boldsymbol{\beta}, \boldsymbol{h}_{y}, \mathcal{D}^{T}$

3. $\boldsymbol{\xi}_{y} \mid \boldsymbol{\mu}_{y}, \boldsymbol{J}_{y}, \boldsymbol{\beta}, \boldsymbol{h}_{y}, \sigma_{\xi}^{2}, \mathcal{D}^{T}$

4. $\boldsymbol{h}_{y} \mid \boldsymbol{\mu}_{y}, \boldsymbol{\xi}_{y}, \boldsymbol{J}_{y}, \mu_{h}, \phi_{h}, \sigma_{h}^{2}, \beta, \mathcal{D}^{T}$

5. $\mu_{y}, \phi_{\mu}, \sigma_{\mu}^{2}, \mu_{h}, \phi_{h}, \sigma_{h}^{2}, \boldsymbol{\lambda}, \sigma_{\xi}^{2}, \boldsymbol{\beta}, \boldsymbol{\beta}_{h} \mid \boldsymbol{\mu}_{y}, \boldsymbol{h}_{y}, \boldsymbol{\xi}_{y}, \boldsymbol{J}_{y}, \mathcal{D}^{T}$

where $\mathcal{D}^{T}$ denotes the information set available at time $T$. The last block is further broken down into 10 separate sub-blocks, one for each element of the parameter vector. We next describe in details the steps of the Gibbs sampler algorithm.

\section{A.3.1 $\boldsymbol{\mu}_{y} \mid \boldsymbol{h}_{y}, \boldsymbol{\xi}_{y}, \boldsymbol{J}_{y}, \mu_{y}, \phi_{\mu}, \sigma_{\mu}^{2}, \boldsymbol{\beta}, \mathcal{D}^{T}$}

Start by rewriting the observation equation in (A.1) as follows:

$$
\boldsymbol{y}^{\star}=\boldsymbol{X}_{\mu} \boldsymbol{\mu}_{y}+\varepsilon_{y} \quad \varepsilon_{y} \sim \mathcal{N}\left(\mathbf{0}, \boldsymbol{\Sigma}_{y}\right)
$$

where

$$
\begin{gathered}
\boldsymbol{y}^{\star}=\left[\begin{array}{c}
y_{1}-\xi_{y 1} J_{y 1}-\boldsymbol{\beta}^{\prime} \boldsymbol{X}_{1} \\
\vdots \\
y_{T}-\xi_{y T} J_{y T}-\boldsymbol{\beta}^{\prime} \boldsymbol{X}_{T}
\end{array}\right], \\
\boldsymbol{X}_{\mu}=\left[\begin{array}{ccc}
1 & & \\
& \ddots & \\
& & 1
\end{array}\right] \quad \boldsymbol{\mu}_{y}=\left[\begin{array}{c}
\mu_{y 1} \\
\vdots \\
\mu_{y T}
\end{array}\right] \quad \boldsymbol{\varepsilon}_{y}=\left[\begin{array}{c}
\varepsilon_{y 1} \\
\vdots \\
\varepsilon_{y T}
\end{array}\right],
\end{gathered}
$$

and

$$
\boldsymbol{\Sigma}_{y}=\left[\begin{array}{lll}
e^{h_{y 1}} & & \\
& \ddots & \\
& & e^{h_{y T}}
\end{array}\right]
$$

Next, combine the state equation for $\boldsymbol{\mu}_{y}$ in (A.2) with the initial condition in (A.6) into:

$$
\boldsymbol{H}_{\mu} \boldsymbol{\mu}_{y}=\widetilde{\boldsymbol{\delta}}_{\mu}+\varepsilon_{\mu} \quad \varepsilon_{\mu} \sim \mathcal{N}\left(\mathbf{0}, \boldsymbol{\Sigma}_{\mu}\right)
$$


where

$$
\boldsymbol{H}_{\mu}=\left[\begin{array}{ccccc}
1 & 0 & \ldots & \ldots & 0 \\
-\phi_{\mu} & 1 & 0 & \ldots & 0 \\
\vdots & \vdots & \vdots & \ddots & \vdots \\
0 & \ldots & 0 & -\phi_{\mu} & 1
\end{array}\right] \quad \widetilde{\boldsymbol{\delta}}_{\mu}=\left[\begin{array}{c}
\mu_{y} \\
\left(1-\phi_{\mu}\right) \mu_{y} \\
\vdots \\
\left(1-\phi_{\mu}\right) \mu_{y}
\end{array}\right]
$$

and

$$
\boldsymbol{\Sigma}_{\mu}=\left[\begin{array}{cccc}
\frac{\sigma_{\mu}^{2}}{\left(1-\phi_{\mu}^{2}\right)} & & & \\
& \sigma_{\mu}^{2} & & \\
& & \ddots & \\
& & & \sigma_{\mu}^{2}
\end{array}\right]
$$

It is easy to show that

$$
\boldsymbol{\mu}_{y}=\boldsymbol{\delta}_{\mu}+\boldsymbol{H}_{\mu}^{-1} \varepsilon_{\mu}
$$

where $\boldsymbol{\delta}_{\mu}=\boldsymbol{H}_{\mu}^{-1} \widetilde{\boldsymbol{\delta}}_{\mu}$. It follows that

$$
\boldsymbol{\mu}_{y} \sim \mathcal{N}\left(\boldsymbol{\delta}_{\mu}, \boldsymbol{H}_{\mu}^{-1} \boldsymbol{\Sigma}_{\mu}\left(\boldsymbol{H}_{\mu}^{-1}\right)^{\prime}\right)
$$

or

$$
\boldsymbol{\mu}_{y} \sim \mathcal{N}\left(\boldsymbol{\delta}_{\mu},\left(\boldsymbol{H}_{\mu}^{\prime} \boldsymbol{\Sigma}_{\mu}^{-1} \boldsymbol{H}_{\mu}\right)^{-1}\right)
$$

Finally, combining (A.14) and (A.23) leads to the following posterior:

$$
\boldsymbol{\mu}_{y} \mid \boldsymbol{h}_{y}, \boldsymbol{\xi}_{y}, \boldsymbol{J}_{y}, \mu_{y}, \phi_{\mu}, \sigma_{\mu}^{2}, \boldsymbol{\beta}, \mathcal{D}^{T} \sim \mathcal{N}\left(\overline{\boldsymbol{\mu}}, \overline{\boldsymbol{V}}_{\mu}\right),
$$

where

$$
\begin{aligned}
\overline{\boldsymbol{V}}_{\mu} & =\left[\boldsymbol{H}_{\mu}^{\prime} \boldsymbol{\Sigma}_{\mu}^{-1} \boldsymbol{H}_{\mu}+\boldsymbol{X}_{\mu}^{\prime} \boldsymbol{\Sigma}_{y}^{-1} \boldsymbol{X}_{\mu}\right]^{-1} \\
\overline{\boldsymbol{\mu}} & =\overline{\boldsymbol{V}}_{\mu}\left[\left(\boldsymbol{H}_{\mu}^{\prime} \boldsymbol{\Sigma}_{\mu}^{-1} \boldsymbol{H}_{\mu}\right) \boldsymbol{\delta}_{\mu}+\boldsymbol{X}_{\mu}^{\prime} \boldsymbol{\Sigma}_{y}^{-1} \boldsymbol{y}^{\star}\right] .
\end{aligned}
$$




\section{A.3.2 $\boldsymbol{J}_{y} \mid \boldsymbol{\mu}_{y}, \boldsymbol{\xi}_{y}, \boldsymbol{\beta}, \boldsymbol{h}_{y}, \mathcal{D}^{T}$}

It is easy to show that for any given $t \in[1, T]$

$$
\begin{aligned}
\operatorname{Pr}\left(J_{y t}=1 \mid \mu_{y t}, \xi_{y t}, \boldsymbol{\beta}, \boldsymbol{\lambda}, \boldsymbol{X}_{t}, \boldsymbol{X}_{t}^{J}, h_{t}, \mathcal{D}^{T}\right) & \propto p\left(y_{t} \mid \mu_{y t}, \xi_{y t}, J_{y t}=1, \boldsymbol{\beta}, \boldsymbol{X}_{t}, h_{y t}\right) \\
& \times \operatorname{Pr}\left(J_{y t}=1 \mid \boldsymbol{X}_{t}^{J}, \boldsymbol{\lambda}\right)
\end{aligned}
$$

where

$$
p\left(y_{t} \mid \mu_{y t}, \xi_{y t}, J_{y t}=1, \boldsymbol{\beta}, \boldsymbol{X}_{t}, h_{y t}\right) \sim \mathcal{N}\left(y_{t} \mid \mu_{y t}+\xi_{y t}+\boldsymbol{\beta}^{\prime} \boldsymbol{X}_{t}, e^{h_{y t}}\right),
$$

and $\operatorname{Pr}\left(J_{y t}=1 \mid \boldsymbol{X}_{t}^{J}, \boldsymbol{\lambda}\right)=\Phi\left(\boldsymbol{\lambda}^{\prime} \boldsymbol{X}_{t}^{J}\right)$, while

$$
\begin{aligned}
\operatorname{Pr}\left(J_{y t}=0 \mid \mu_{y t}, \xi_{y t}, \boldsymbol{\beta}, \boldsymbol{\lambda}, \boldsymbol{X}_{t}, \boldsymbol{X}_{t}^{J}, h_{y t}, \mathcal{D}^{T}\right) & \propto p\left(y_{t} \mid \mu_{y t}, \xi_{y t}, J_{y t}=0, \boldsymbol{\beta}, \boldsymbol{X}_{t}, h_{y t}\right) \\
& \times \operatorname{Pr}\left(J_{y t}=0 \mid \boldsymbol{X}_{t}^{J}, \boldsymbol{\lambda}\right),
\end{aligned}
$$

where

$$
p\left(y_{t} \mid \mu_{y t}, \xi_{y t}, J_{y t}=0, \boldsymbol{\beta}, \boldsymbol{X}_{t}, h_{y t}\right) \sim \mathcal{N}\left(y_{t} \mid \mu_{y t}+\boldsymbol{\beta}^{\prime} \boldsymbol{X}_{t}, e^{h_{y t}}\right)
$$

and $\operatorname{Pr}\left(J_{y t}=0 \mid \boldsymbol{X}_{t}^{J}, \boldsymbol{\lambda}\right)=1-\Phi\left(\boldsymbol{\lambda}^{\prime} \boldsymbol{X}_{t}^{J}\right)$.

\section{A.3.3 $\boldsymbol{\xi}_{y} \mid \boldsymbol{\mu}_{y}, \boldsymbol{J}_{y}, \boldsymbol{\beta}, \boldsymbol{h}_{y}, \sigma_{\xi}^{2}, \mathcal{D}^{T}$}

Start by noting that when $J_{y t}=0, \xi_{y t} \mid J_{y t}=0, \mathcal{D}^{T} \sim \mathcal{N}\left(0, \underline{\sigma}_{\xi}^{2}\right)$. In other words, when $J_{y t}=0$ we rely on the prior distribution of $\xi_{y t}$ in (A.5). In contrast, when $J_{y t}=1$, it is possible to rewrite the observation equation of the model in (A.1) as

$$
y_{t}-\mu_{y t}-\boldsymbol{\beta}^{\prime} \boldsymbol{X}_{t}=\xi_{y t}+\varepsilon_{y t}, \quad \varepsilon_{y t} \sim \mathcal{N}\left(0, e^{h_{y t}}\right) .
$$

Combining (A.30) with (A.5) leads to

$$
\xi_{y t} \mid \mu_{y t}, J_{y t}=1, \boldsymbol{\beta}, h_{y t}, \sigma_{\xi}^{2}, \mathcal{D}^{T} \sim \mathcal{N}\left(\bar{\mu}_{\xi_{y t}}, \bar{\sigma}_{\xi_{y t}}^{2}\right)
$$


where

$$
\begin{aligned}
\bar{\sigma}_{\xi_{y t}}^{2} & =\left(\underline{\sigma}_{\xi}^{-2}+e^{-h_{y t}}\right)^{-1} \\
\bar{\mu}_{\xi_{y t}} & =\bar{\sigma}_{\xi_{y t}}^{2}\left(e^{-h_{y t}}\left(y_{t}-\mu_{y t}-\boldsymbol{\beta}^{\prime} \boldsymbol{X}_{t}\right)\right) .
\end{aligned}
$$

\section{A.3.4 $\boldsymbol{h}_{y} \mid \boldsymbol{\mu}_{y}, \boldsymbol{\xi}_{y}, \boldsymbol{J}_{y}, \mu_{h}, \phi_{h}, \sigma_{h}^{2}, \boldsymbol{\beta}, \mathcal{D}^{T}$}

Combining the state equation for $h_{y t}$ in (A.3) with the initial condition for $h_{y 1}$ in (A.7), we have:

$$
\boldsymbol{H}_{h} \boldsymbol{h}_{y}=\widetilde{\boldsymbol{\delta}}_{h}+\varepsilon_{h}, \quad \varepsilon_{h} \sim \mathcal{N}\left(\mathbf{0}, \boldsymbol{\Sigma}_{h}\right)
$$

where

$$
\boldsymbol{H}_{h}=\left[\begin{array}{cccccc}
1 & 0 & \ldots & \ldots & \ldots & 0 \\
-\phi_{h} & 1 & 0 & \ldots & \ldots & 0 \\
\vdots & \vdots & \vdots & \ddots & \vdots & \vdots \\
0 & \ldots & \ldots & 0 & -\phi_{h} & 1
\end{array}\right], \quad \widetilde{\boldsymbol{\delta}}_{h}=\left[\begin{array}{c}
\mu_{h}+\frac{\boldsymbol{\beta}_{h}^{\prime} \boldsymbol{X}_{1}}{\left(1-\phi_{h}\right)} \\
\left(1-\phi_{h}\right) \mu_{h}+\boldsymbol{\beta}_{h}^{\prime} \boldsymbol{X}_{2} \\
\ldots \\
\ldots \\
\left(1-\phi_{h}\right) \mu_{h}+\boldsymbol{\beta}_{h}^{\prime} \boldsymbol{X}_{T}
\end{array}\right]
$$

and

$$
\boldsymbol{\Sigma}_{h}=\left[\begin{array}{cccc}
\frac{\sigma_{h}^{2}}{\left(1-\phi_{h}^{2}\right)} & & & \\
& \sigma_{h}^{2} & & \\
& & \ddots & \\
& & & \sigma_{h}^{2}
\end{array}\right]
$$

This leads to

$$
\boldsymbol{h}_{y} \sim \mathcal{N}\left(\boldsymbol{\delta}_{h},\left(\boldsymbol{H}_{h}^{\prime} \boldsymbol{\Sigma}_{h}^{-1} \boldsymbol{H}_{h}\right)^{-1}\right)
$$

where $\boldsymbol{\delta}_{h}=\boldsymbol{H}_{h}^{-1} \widetilde{\boldsymbol{\delta}}_{h}$. Next, note that the observation equation is a non-linear function of $\boldsymbol{h}_{y}$, so we follow Chan and Grant (2016a) and first approximate it with a Gaussian density in $\boldsymbol{h}_{y}$. Recall from (A.14) that

$$
\boldsymbol{y}^{\star} \mid \boldsymbol{\mu}_{y}, \boldsymbol{h}_{y}, \boldsymbol{J}_{y}, \boldsymbol{\xi}_{y}, \boldsymbol{\beta} \sim \mathcal{N}\left(\boldsymbol{\mu}_{y}, \boldsymbol{\Sigma}_{y}\right)
$$


A second-order Taylor expansion of (A.37) around $\widetilde{\boldsymbol{h}}_{y}$, the mode of the posterior (see Chan and Grant (2016a) for additional details), leads to the following approximation:

$$
\begin{aligned}
\ln p\left(\boldsymbol{y}^{\star} \mid \boldsymbol{\mu}_{y}, \boldsymbol{h}_{y}, \boldsymbol{J}_{y}, \boldsymbol{\xi}_{y}, \boldsymbol{\beta}\right) & \approx \ln p\left(\boldsymbol{y}^{\star} \mid \boldsymbol{\mu}_{y}, \widetilde{\boldsymbol{h}}_{y}, \boldsymbol{J}_{y}, \boldsymbol{\xi}_{y}, \boldsymbol{\beta}\right)+\left(\boldsymbol{h}_{y}-\widetilde{\boldsymbol{h}}_{y}\right)^{\prime} \boldsymbol{f} \\
& -\frac{1}{2}\left(\boldsymbol{h}_{y}-\widetilde{\boldsymbol{h}}_{y}\right)^{\prime} \boldsymbol{G}\left(\boldsymbol{h}_{y}-\widetilde{\boldsymbol{h}}_{y}\right)
\end{aligned}
$$

where $\boldsymbol{f}$ is $T \times 1$ vector of gradients and $\boldsymbol{G}$ is $T \times T$ matrix containing the elements of the negative Hessian, while $\widetilde{\boldsymbol{h}}_{y}$ denotes the mode of $\ln p\left(\boldsymbol{y}^{\star} \mid \boldsymbol{\mu}_{y}, \boldsymbol{h}_{y}, \boldsymbol{J}_{y}, \boldsymbol{\xi}_{y}, \boldsymbol{\beta}\right)$. In particular,

$$
\boldsymbol{f}=\left[\begin{array}{c}
f_{1} \\
\vdots \\
f_{T}
\end{array}\right], \quad \boldsymbol{G}=\left[\begin{array}{lll}
G_{11} & & \\
& \ddots & \\
& & G_{T T}
\end{array}\right]
$$

with

$$
f_{t}=\left.\frac{\partial \ln p\left(y_{t}^{\star} \mid \mu_{y t}, h_{y t}, J_{y t}, \xi_{y t}, \boldsymbol{\beta}\right)}{\partial h_{y t}}\right|_{h_{y t}=\tilde{h}_{y t}},
$$

and

$$
G_{t t}=-\left.\frac{\partial^{2} \ln p\left(y_{t}^{\star} \mid \mu_{y t}, h_{y t}, J_{y t}, \xi_{y t}, \boldsymbol{\beta}\right)}{\partial h_{y t}^{2}}\right|_{h_{y t}=\tilde{h}_{y t}},
$$

with

$$
\begin{aligned}
f_{t} & =-\frac{1}{2}+\frac{1}{2} e^{-h_{y t}}\left(y_{t}^{\star}-\mu_{y t}\right)^{2}, \\
G_{t t} & =-\frac{1}{2} e^{-h_{y t}}\left(y_{t}^{\star}-\mu_{y t}\right)^{2} .
\end{aligned}
$$

Some additional algebra leads to

$$
\begin{aligned}
\ln p\left(\boldsymbol{y}^{\star} \mid \boldsymbol{\mu}_{y}, \boldsymbol{h}_{y}, \boldsymbol{J}_{y}, \boldsymbol{\xi}_{y}, \boldsymbol{\beta}\right) & \propto \boldsymbol{h}_{y}^{\prime} \boldsymbol{f}-\frac{1}{2} \boldsymbol{h}_{y}^{\prime} \boldsymbol{G} \boldsymbol{h}_{y}+\boldsymbol{h}_{y}^{\prime} \boldsymbol{G} \widetilde{\boldsymbol{h}}_{y} \\
& \propto-\frac{1}{2}\left(\boldsymbol{h}_{y}^{\prime} \boldsymbol{G} \boldsymbol{h}_{y}-2 \boldsymbol{h}_{y}^{\prime}\left(\boldsymbol{f}+\boldsymbol{G} \widetilde{\boldsymbol{h}}_{y}\right)\right) .
\end{aligned}
$$

Combining (A.43) with (A.36) leads to the following posterior for $\boldsymbol{h}_{y}$ :

$$
\boldsymbol{h}_{y} \mid \boldsymbol{\mu}_{y}, \boldsymbol{\xi}_{y}, \boldsymbol{J}_{y}, \mu_{h}, \phi_{h}, \sigma_{h}^{2}, \boldsymbol{\beta}, \mathcal{D}^{T} \sim \mathcal{N}\left(\boldsymbol{K}_{h}^{-1} \boldsymbol{k}_{h}, \boldsymbol{K}_{h}^{-1}\right),
$$


where

$$
\begin{aligned}
\boldsymbol{K}_{h} & =\boldsymbol{H}_{h}^{\prime} \boldsymbol{\Sigma}_{h}^{-1} \boldsymbol{H}_{h}+\boldsymbol{G}, \\
\boldsymbol{k}_{h} & =\boldsymbol{H}_{h}^{\prime} \boldsymbol{\Sigma}_{h}^{-1} \boldsymbol{H}_{h} \boldsymbol{\delta}_{h}+\boldsymbol{f}+\boldsymbol{G} \widetilde{\boldsymbol{h}}_{y} .
\end{aligned}
$$

A.3.5 $\mu_{y}, \phi_{\mu}, \sigma_{\mu}^{2}, \mu_{h}, \phi_{h}, \sigma_{h}^{2}, \boldsymbol{\lambda}, \sigma_{\xi}^{2}, \boldsymbol{\beta}, \boldsymbol{\beta}_{h} \mid \boldsymbol{\mu}_{y}, \boldsymbol{h}_{y}, \boldsymbol{\xi}_{y}, \boldsymbol{J}_{y}, \mathcal{D}^{T}$

We break the posterior into 10 separate blocks:

- $\mu_{y} \mid \boldsymbol{\mu}_{y}, \phi_{\mu}, \sigma_{\mu}^{2}, \mathcal{D}^{T}$ :

Start by combining (A.2) and (A.6) and rewriting them as:

$$
\boldsymbol{Z}_{\mu}=\boldsymbol{X}_{\mu} \mu_{y}+\boldsymbol{\epsilon}_{\mu} \quad \boldsymbol{\epsilon}_{\mu} \sim \mathcal{N}\left(\mathbf{0}, \boldsymbol{\Sigma}_{\mu}\right)
$$

where

$$
\boldsymbol{Z}_{\mu}=\left[\begin{array}{c}
\mu_{y 1} \\
\mu_{y 2}-\phi_{\mu} \mu_{y 1} \\
\vdots \\
\mu_{y T}-\phi_{\mu} \mu_{y T-1}
\end{array}\right], \quad \boldsymbol{X}_{\mu}=\left[\begin{array}{c}
1 \\
\left(1-\phi_{\mu}\right) \\
\vdots \\
\left(1-\phi_{\mu}\right)
\end{array}\right]
$$

Combining (A.47) with the prior for $\mu_{y}$ in (A.8) leads to

$$
\mu_{y} \mid \boldsymbol{\mu}_{y}, \phi_{\mu}, \sigma_{\mu}^{2}, \mathcal{D}^{T} \sim \mathcal{N}\left(\bar{\mu}_{y}, \bar{V}_{\mu_{y}}\right)
$$

where

$$
\bar{V}_{\mu_{y}}=\left[\underline{V}_{\mu_{y}}^{-1}+\boldsymbol{X}_{\mu}^{\prime} \boldsymbol{\Sigma}_{\mu}^{-1} \boldsymbol{X}_{\mu}\right]^{-1}
$$

and

$$
\bar{\mu}_{y}=\bar{V}_{\mu_{y}}\left[\underline{V}_{\mu_{y}} \underline{\mu}_{y 0}+\boldsymbol{X}_{\mu}^{\prime} \boldsymbol{\Sigma}_{\mu}^{-1} \boldsymbol{Z}_{\mu}\right] .
$$

- $\phi_{\mu} \mid \boldsymbol{\mu}_{y}, \mu_{y}, \sigma_{\mu}^{2}, \mathcal{D}^{T}$ : 
Following Kim et al. (1998), we start by obtaining a candidate draw from the following distribution:

$$
\phi_{\mu}^{\star} \sim \mathcal{N}\left(\bar{\phi}_{\mu}, \bar{V}_{\mu}\right) \times I\left(\left|\phi_{\mu}\right|<1\right)
$$

where

$$
\begin{gathered}
\bar{V}_{\phi_{\mu}}=\left(\underline{V}_{\phi_{\mu}}^{-1}+\frac{\boldsymbol{X}_{\phi_{\mu}}^{\prime} \boldsymbol{X}_{\phi_{\mu}}}{\sigma_{\mu}^{2}}\right)^{-1}, \\
\bar{\phi}_{\mu}=\bar{V}_{\phi_{\mu}}\left(\underline{V}_{\phi_{\mu}}^{-1} \underline{\mu}_{\mu_{0}}+\frac{\boldsymbol{X}_{\phi_{\mu}}^{\prime} \boldsymbol{Z}_{\phi_{\mu}}}{\sigma_{\mu}^{2}}\right)
\end{gathered}
$$

and where

$$
\boldsymbol{Z}_{\phi_{\mu}}=\left[\begin{array}{c}
\mu_{y 2}-\mu_{y} \\
\vdots \\
\mu_{y T}-\mu_{y}
\end{array}\right], \quad \boldsymbol{X}_{\phi_{\mu}}=\left[\begin{array}{c}
\mu_{y 1}-\mu_{y} \\
\vdots \\
\mu_{y T-1}-\mu_{y}
\end{array}\right]
$$

Next, if the draw is retained (i.e., satisfies the stationarity restriction), we accept $\phi_{\mu}^{*}$ with probability $e^{\left(g\left(\phi_{\mu}^{*}\right)-g\left(\phi_{\mu}^{o l d}\right)\right)}$ where $\phi_{\mu}^{\text {old }}$ is the retained draw from the previous iteration of the Gibbs sampler, and

$$
g\left(\phi_{\mu}\right)=\ln p\left(\phi_{\mu}\right)-\frac{1}{2} \ln \left(\frac{\sigma_{\mu}^{2}}{1-\phi_{\mu}^{2}}\right)-\frac{\left(1-\phi_{\mu}^{2}\right)}{2 \sigma_{\mu}^{2}}\left(\mu_{y 1}-\mu_{y}\right)^{2},
$$

with $p\left(\phi_{\mu}\right)$ denoting the prior of $\phi_{\mu}$ from (A.8).

- $\sigma_{\mu}^{2} \mid \boldsymbol{\mu}_{y}, \mu_{y}, \phi_{\mu}, \mathcal{D}^{T}$ :

The posterior for $\sigma_{\mu}^{2}$ is readily available, and is given by:

$$
\sigma_{\mu}^{2} \mid \boldsymbol{\mu}_{y}, \mu_{y}, \phi_{\mu}, \mathcal{D}^{T} \sim \mathcal{I} \mathcal{G}\left(\underline{\nu}_{\mu}+\frac{T}{2}, \bar{S}_{\mu}\right)
$$

where

$$
\bar{S}_{\mu}=\underline{S}_{\mu}+\frac{1}{2}\left[\left(1-\phi_{\mu}^{2}\right)\left(\mu_{y 1}-\mu_{y}\right)^{2}+\sum_{t=1}^{T-1}\left(\mu_{y t+1}-\mu_{y}-\phi_{\mu}\left(\mu_{y t}-\mu_{y}\right)\right)^{2}\right] .
$$


- $\mu_{h} \mid \boldsymbol{h}_{y}, \phi_{h}, \sigma_{h}^{2}, \boldsymbol{\beta}_{h} \mathcal{D}^{T}:$

We combine (A.3) and (A.7) into

$$
\boldsymbol{Z}_{h}=\boldsymbol{X}_{h} \mu_{h}+\varepsilon_{h} \quad \varepsilon_{h} \sim \mathcal{N}\left(\mathbf{0}, \boldsymbol{\Sigma}_{h}\right),
$$

where

$$
\boldsymbol{X}_{h}=\left[\begin{array}{c}
1 \\
1-\phi_{h} \\
\vdots \\
1-\phi_{h}
\end{array}\right], \quad \boldsymbol{Z}_{h}=\left[\begin{array}{c}
h_{y 1}-\frac{\boldsymbol{\beta}_{h}^{\prime} \boldsymbol{X}_{1}^{h}}{1-\phi_{h}} \\
h_{y 2}-\phi_{h} h_{y 1}-\boldsymbol{\beta}_{h}^{\prime} \boldsymbol{X}_{2}^{h} \\
\vdots \\
h_{y T}-\phi_{h} h_{y T-1}-\boldsymbol{\beta}_{h}^{\prime} \boldsymbol{X}_{T}^{h}
\end{array}\right]
$$

Next, combine (A.59) with the prior for $\mu_{h}$ in (A.9) to get

$$
\mu_{h} \mid \boldsymbol{h}_{y}, \phi_{h}, \sigma_{h}^{2}, \boldsymbol{\beta}_{h}, \mathcal{D}^{T} \sim \mathcal{N}\left(\bar{\mu}_{h}, \bar{V}_{\mu_{h}}\right),
$$

where

$$
\bar{V}_{\mu_{h}}=\left[\underline{V}_{\mu_{h}}^{-1}+\boldsymbol{X}_{h}^{\prime} \boldsymbol{\Sigma}_{h}^{-1} \boldsymbol{X}_{h}\right]^{-1},
$$

and

$$
\bar{\mu}_{h}=\bar{V}_{\mu_{h}}\left[\underline{V}_{\mu_{h}}^{-1} \underline{\mu}_{h 0}+\boldsymbol{X}_{h}^{\prime} \boldsymbol{\Sigma}_{h}^{-1} \boldsymbol{Z}_{h}\right] .
$$

- $\phi_{h} \mid \boldsymbol{h}_{y}, \mu_{h}, \sigma_{h}^{2}, \boldsymbol{\beta}_{h}, \mathcal{D}^{T}$ :

As with $\phi_{\mu}$, we follow Kim et al. (1998) and first obtain a candidate draw from the following distribution:

$$
\phi_{h}^{\star} \sim \mathcal{N}\left(\bar{\phi}_{h}, \bar{V}_{h}\right) \times I\left(\left|\phi_{h}\right|<1\right),
$$

where

$$
\bar{V}_{\phi_{h}}=\left(\underline{V}_{\phi_{h}}^{-1}+\frac{\boldsymbol{X}_{\phi_{h}}^{\prime} \boldsymbol{X}_{\phi_{h}}}{\sigma_{h}^{2}}\right)^{-1},
$$




$$
\bar{\phi}_{h}=\bar{V}_{\phi_{h}}\left(\underline{V}_{\phi_{h}}^{-1} \underline{\phi}_{h_{0}}+\frac{\boldsymbol{X}_{\phi_{h}}^{\prime} \boldsymbol{Z}_{\phi_{h}}}{\sigma_{h}^{2}}\right)
$$

and where

$$
\boldsymbol{Z}_{\phi_{h}}=\left[\begin{array}{c}
h_{y 2}-\mu_{h}-\boldsymbol{\beta}_{h}^{\prime} \boldsymbol{X}_{2}^{h} \\
\vdots \\
h_{y T}-\mu_{h}-\boldsymbol{\beta}_{h}^{\prime} \boldsymbol{X}_{T}^{h}
\end{array}\right], \quad \boldsymbol{X}_{\phi_{h}}=\left[\begin{array}{c}
h_{y 1}-\mu_{h} \\
\vdots \\
h_{y T-1}-\mu_{h}
\end{array}\right] .
$$

Next, if the draw is retained (i.e., satisfies the stationarity restriction), we accept $\phi_{h}^{*}$ with probability $e^{\left(g\left(\phi_{h}^{*}\right)-g\left(\phi_{h}^{\text {old }}\right)\right)}$ where $\phi_{h}^{\text {old }}$ is the retained draw from the previous iteration of the Gibbs sampler, and

$$
g\left(\phi_{h}\right)=\ln p\left(\phi_{h}\right)-\frac{1}{2} \ln \left(\frac{\sigma_{h}^{2}}{1-\phi_{h}^{2}}\right)-\frac{\left(1-\phi_{h}^{2}\right)}{2 \sigma_{h}^{2}}\left(h_{y 1}-\mu_{h}-\frac{\boldsymbol{\beta}_{h}^{\prime} \boldsymbol{X}_{1}^{h}}{1-\phi_{h}}\right)^{2}
$$

with $p\left(\phi_{h}\right)$ denoting the prior of $\phi_{h}$.

- $\sigma_{h}^{2} \mid \boldsymbol{h}_{y}, \mu_{h}, \phi_{h}, \beta_{h}, \mathcal{D}^{T}$ :

The posterior for $\sigma_{h}^{2}$ is readily available, and is given by

$$
\sigma_{h}^{2} \mid \boldsymbol{h}_{y}, \mu_{h}, \phi_{h}, \beta_{h}, \mathcal{D}^{T} \sim \mathcal{I} \mathcal{G}\left(\underline{\nu}_{h}+\frac{T}{2}, \bar{S}_{h}\right)
$$

where

$$
\begin{aligned}
\bar{S}_{h}=\underline{S}_{h} & +\frac{1}{2}\left[\left(1-\phi_{h}^{2}\right)\left(h_{y 1}-\mu_{h}-\frac{\boldsymbol{\beta}_{h}^{\prime} \boldsymbol{X}_{1}^{h}}{1-\phi_{h}}\right)^{2}\right. \\
& \left.+\sum_{t=1}^{T-1}\left(h_{y t+1}-\mu_{h}-\phi_{h}\left(h_{y t}-\mu_{h}\right)-\boldsymbol{\beta}_{h}^{\prime} \boldsymbol{X}_{t+1}^{h}\right)^{2}\right]
\end{aligned}
$$

- $\boldsymbol{\beta}_{h} \mid \boldsymbol{h}_{y}, \mu_{h}, \phi_{h}, \sigma_{h}^{2}, \mathcal{D}^{T}$ :

Start by rewriting (A.3) as follows

$$
\boldsymbol{Z}_{\boldsymbol{\beta}_{h}}=\boldsymbol{X}_{\boldsymbol{\beta}_{h}} \boldsymbol{\beta}_{h}+\varepsilon_{h} \quad \varepsilon_{h} \sim \mathcal{N}\left(\mathbf{0}, \boldsymbol{\Sigma}_{h}\right)
$$


where

$$
\boldsymbol{Z}_{\boldsymbol{\beta}_{h}}=\left[\begin{array}{c}
h_{y 1}-\mu_{h} \\
h_{y 2}-\mu_{h}-\phi_{h}\left(h_{y 1}-\mu_{h}\right) \\
\vdots \\
h_{y T}-\mu_{h}-\phi_{h}\left(h_{y T-1}-\mu_{h}\right)
\end{array}\right], \quad \boldsymbol{X}_{\beta_{h}}=\left[\begin{array}{c}
\left(1-\phi_{h}\right)^{-1} \boldsymbol{X}_{1}^{h \prime} \\
\boldsymbol{X}_{2}^{h \prime} \\
\vdots \\
\boldsymbol{X}_{T}^{h \prime}
\end{array}\right] .
$$

Combing (A.71) with the prior for $\boldsymbol{\beta}_{h}$ in (A.13) leads to the following posterior distribution:

$$
\boldsymbol{\beta}_{h} \mid \boldsymbol{h}_{y}, \mu_{h}, \phi_{h}, \sigma_{h}^{2}, \mathcal{D}^{T} \sim \mathcal{N}\left(\overline{\boldsymbol{\beta}}_{h}, \overline{\boldsymbol{V}}_{\boldsymbol{\beta}_{h}}\right)
$$

where

$$
\overline{\boldsymbol{V}}_{\boldsymbol{\beta}_{h}}=\left(\underline{\boldsymbol{V}}_{\boldsymbol{\beta}_{h}}^{-1}+\boldsymbol{X}_{\boldsymbol{\beta}_{h}}^{\prime} \boldsymbol{\Sigma}_{h}^{-1} \boldsymbol{X}_{\boldsymbol{\beta}_{h}}\right)^{-1}
$$

and

$$
\overline{\boldsymbol{\beta}}_{h}=\overline{\boldsymbol{V}}_{\boldsymbol{\beta}_{h}}\left(\underline{\boldsymbol{V}}_{\boldsymbol{\beta}_{h}}^{-1} \underline{\boldsymbol{\beta}}_{h_{0}}+\boldsymbol{X}_{\beta_{h}}^{\prime} \boldsymbol{\Sigma}_{h}^{-1} \boldsymbol{Z}_{\boldsymbol{\beta}_{h}}\right) .
$$

- $\boldsymbol{\lambda} \mid \boldsymbol{W}, \mathcal{D}^{T}$ and $\boldsymbol{W} \mid \boldsymbol{\lambda}, \boldsymbol{J}_{y}, \mathcal{D}^{T}$ :

We follow Albert and Chib (1993) and to simplify the computations introduce the auxiliary latent state variable $W_{t}, t=1, \ldots, T$. We proceed by first rewriting the stochastic process of the jump intensity in (A.4) as

$$
J_{y t+1}= \begin{cases}1 & \text { if } W_{t+1}>0 \\ 0 & \text { if } W_{t+1} \leq 0\end{cases}
$$

where

$$
W_{t+1}=\boldsymbol{\lambda}^{\prime} \boldsymbol{X}_{t+1}^{J}+\varepsilon_{W t+1}, \quad \varepsilon_{W t+1} \sim \mathcal{N}(0,1)
$$

or, more compactly,

$$
\boldsymbol{W}=\boldsymbol{X}^{J} \boldsymbol{\lambda}+\varepsilon_{W}, \quad \varepsilon_{W} \sim \mathcal{N}\left(\mathbf{0}, \boldsymbol{I}_{T}\right)
$$


where

$$
\boldsymbol{X}^{J}=\left[\begin{array}{c}
\boldsymbol{X}_{1}^{J \prime} \\
\vdots \\
\boldsymbol{X}_{T}^{J \prime}
\end{array}\right], \quad \boldsymbol{W}=\left[\begin{array}{c}
W_{1} \\
\vdots \\
W_{T}
\end{array}\right] .
$$

The posterior of $\boldsymbol{\lambda}$ is readily available and is given by

$$
\boldsymbol{\lambda} \mid \boldsymbol{W}, \mathcal{D}^{T} \sim \mathcal{N}\left(\overline{\boldsymbol{\mu}}_{\lambda}, \overline{\boldsymbol{V}}_{\lambda}\right)
$$

where

$$
\overline{\boldsymbol{V}}_{\lambda}=\left[\underline{\boldsymbol{V}}_{\lambda}^{-1}+\boldsymbol{X}^{J \prime} \boldsymbol{X}^{J}\right]^{-1},
$$

and

$$
\overline{\boldsymbol{\mu}}_{\lambda}=\overline{\boldsymbol{V}}_{\lambda}\left[\underline{\boldsymbol{V}}_{\lambda}^{-1} \underline{\boldsymbol{\mu}}_{\lambda}+\boldsymbol{X}^{J \prime} \boldsymbol{Z}\right]
$$

Turning to the sequence of latent variables $\left\{W_{t}\right\}_{t=1}^{T}$, we have

$$
W_{t} \mid \boldsymbol{\lambda}, J_{y t}, \mathcal{D}^{T} \sim\left\{\begin{array}{l}
\mathcal{T} \mathcal{N}\left(\boldsymbol{\lambda}^{\prime} \boldsymbol{X}_{t}^{J}, 1,0, \infty\right) \text { if } J_{y t}=1 \\
\mathcal{T} \mathcal{N}\left(\boldsymbol{\lambda}^{\prime} \boldsymbol{X}_{t}^{J}, 1,-\infty, 0\right) \text { if } J_{y t}=0
\end{array}\right.
$$

where $\mathcal{T} \mathcal{N}\left(\mu, \sigma^{2}, l b, u b\right)$ denotes a truncated normal distribution with mean $\mu$, variance $\sigma^{2}$, and lower and upper bound $l b, u b$.

- $\sigma_{\xi}^{2} \mid \boldsymbol{\xi}_{y}, \mathcal{D}^{T}$ :

The posterior distribution for $\sigma_{\xi}^{2}$ is readily available and is given by

$$
\sigma_{\xi}^{2} \mid \boldsymbol{\xi}_{y}, \mathcal{D}^{T} \sim \mathcal{I} \mathcal{G}\left(\underline{\nu}_{\xi}+\frac{T}{2}, \bar{S}_{\xi}\right)
$$

where

$$
\bar{S}_{\xi}=\underline{S}_{\xi}+\frac{1}{2} \sum_{t=1}^{T} \xi_{y t}^{2}
$$


- $\boldsymbol{\beta} \mid \boldsymbol{\mu}_{y}, \boldsymbol{h}_{y}, \boldsymbol{J}_{y}, \boldsymbol{\xi}_{y}, \mathcal{D}^{T}:$

Start by rewriting (A.1) as follows:

$$
\boldsymbol{y}^{* *}=\boldsymbol{X} \boldsymbol{\beta}+\varepsilon \quad \varepsilon \sim \mathcal{N}\left(\mathbf{0}, \boldsymbol{\Sigma}_{y}\right),
$$

where

$$
\boldsymbol{y}^{* *}=\left[\begin{array}{c}
y_{1}-\mu_{y 1}-J_{y 1} \xi_{y 1} \\
\vdots \\
y_{T}-\mu_{y T}-J_{y T} \xi_{y T}
\end{array}\right], \quad \boldsymbol{X}=\left[\begin{array}{c}
\boldsymbol{X}_{1}^{\prime} \\
\vdots \\
\boldsymbol{X}_{T}^{\prime}
\end{array}\right]
$$

Next, combine (A.86) with the prior distribution for $\boldsymbol{\beta}$ in (A.12) to obtain:

$$
\boldsymbol{\beta} \mid \boldsymbol{\mu}_{y}, \boldsymbol{h}_{y}, \boldsymbol{J}_{y}, \boldsymbol{\xi}_{y}, \mathcal{D}^{T} \sim \mathcal{N}\left(\overline{\boldsymbol{\beta}}, \overline{\boldsymbol{V}}_{\boldsymbol{\beta}}\right),
$$

where

$$
\overline{\boldsymbol{V}}_{\boldsymbol{\beta}}=\left[\underline{\boldsymbol{V}}_{\boldsymbol{\beta}}^{-1}+\boldsymbol{X}^{\prime} \boldsymbol{\Sigma}_{y}^{-1} \boldsymbol{X}\right]^{-1},
$$

and

$$
\overline{\boldsymbol{\beta}}=\underline{\boldsymbol{V}}_{\boldsymbol{\beta}}\left[\underline{\boldsymbol{V}}_{\boldsymbol{\beta}}^{-1} \underline{\boldsymbol{\beta}}_{0}+\boldsymbol{X}^{\prime} \boldsymbol{\Sigma}_{y}^{-1} \boldsymbol{y}^{* *}\right] .
$$




\section{Appendix B MCMC Convergence and Efficiency}

In this Appendix, we discuss the convergence properties of our MCMC algorithm for both the mean-reverting, stochastic volatility model with jumps described in Section 3.2 and the joint return-cash flow model described in Section 5.1. All results are based on samples of 2,000 retained draws, obtained by sampling a total of 101,000 draws, discarding the first 1,000 draws, and retaining every 20th draw of the post-burn samples.

Table B.1 and Table B.2 report summary statistics of inefficiency factors (IF) for the posterior estimates of all key parameters of the cash flow and return-cash flow models, respectively. Generally speaking, values of the IFs below 20 are taken as indication that the chain has satisfactory mixing properties. As is clear from the entries in both tables, our algorithm shows excellent mixing properties. 


\begin{tabular}{|c|c|c|c|}
\hline \multicolumn{4}{|c|}{ PANEL A: DIVIDENDS } \\
\hline & IF $4 \%$ & IF $8 \%$ & IF $15 \%$ \\
\hline$\mu_{d t}$ & 0.888 & 0.747 & 0.714 \\
\hline$\phi_{\mu}$ & 3.681 & 3.488 & 3.413 \\
\hline$\sigma_{\mu}^{2}$ & 7.054 & 6.337 & 6.454 \\
\hline$\mu_{h}$ & 0.766 & 0.554 & 0.369 \\
\hline$\phi_{h}$ & 0.650 & 0.439 & 0.333 \\
\hline$\sigma_{h}^{2}$ & 0.741 & 0.645 & 0.521 \\
\hline$\sigma_{\xi}^{2}$ & 3.218 & 3.222 & 3.797 \\
\hline$\lambda_{1}$ & 0.857 & 0.897 & 0.998 \\
\hline$\lambda_{2}$ & 1.284 & 1.433 & 1.859 \\
\hline \multicolumn{4}{|c|}{ PANEL B: DIVIDENDS (from 1927) } \\
\hline & IF $4 \%$ & IF $8 \%$ & IF $15 \%$ \\
\hline$\mu_{d t}$ & 1.447 & 1.614 & 1.921 \\
\hline$\phi_{\mu}$ & 3.041 & 3.270 & 3.174 \\
\hline$\sigma_{\mu}^{2}$ & 6.944 & 7.504 & 9.093 \\
\hline$\mu_{h}$ & 0.840 & 0.797 & 0.853 \\
\hline$\phi_{h}$ & 0.741 & 0.612 & 0.409 \\
\hline$\sigma_{h}^{2}$ & 0.616 & 0.474 & 0.336 \\
\hline$\sigma_{\xi}^{2}$ & 1.918 & 1.913 & 1.770 \\
\hline$\lambda_{1}$ & 1.712 & 1.612 & 1.198 \\
\hline$\lambda_{2}$ & 2.667 & 2.509 & 2.087 \\
\hline
\end{tabular}

Table B.1: Inefficiency factors of the model. This table reports the inefficiency factors for the key parameters of the mean-reverting, stochastic volatility model with jumps described in Section 3.2. Panel A reports results for the model using the daily dividend growth series starting in 1973, while Panel B shows estimates using the daily dividend growth series and starting in 1927. For each individual parameter, the inefficiency factor is estimated as $1+2 \sum_{k=1}^{\infty} \rho_{k}$ where $\rho_{k}$ is the $k$ th-order autocorrelation of the chain of retained draws. The estimates use the Newey-West kernel and a bandwidth of $4 \%, 8 \%$, or $15 \%$ of the sample of retained draws. All results are based on a sample of 2,000 retained draws, obtained by sampling a total of 101,000 draws, discarding the first 1,000 , and retaining every 20 th draw of the post-burn sample. 


\begin{tabular}{cccc}
\hline \multicolumn{4}{c}{ Joint returns-cash flow model } \\
\hline & IF 4\% & IF $8 \%$ & IF $15 \%$ \\
$\beta_{1}$ & 0.748 & 0.490 & 0.404 \\
$\beta_{2}$ & 1.321 & 1.289 & 1.340 \\
$\beta_{3}$ & 0.985 & 1.075 & 1.076 \\
$\beta_{4}$ & 0.741 & 0.580 & 0.485 \\
$\mu_{r}$ & 0.985 & 0.849 & 0.660 \\
$\phi_{\mu r}$ & 1.285 & 0.969 & 0.730 \\
& & & \\
$\mu_{h r}$ & 4.367 & 4.195 & 2.582 \\
$\phi_{h r}$ & 1.162 & 1.242 & 1.217 \\
$\gamma_{1}$ & 1.603 & 1.353 & 0.473 \\
$\gamma_{2}$ & 3.454 & 3.215 & 2.010 \\
$\gamma_{3}$ & 14.260 & 13.886 & 11.485 \\
& & & \\
$\lambda_{1}^{r}$ & 3.628 & 3.329 & 2.172 \\
$\lambda_{2}^{r}$ & 1.291 & 1.191 & 1.291 \\
$\lambda_{3}^{r}$ & 1.482 & 1.341 & 1.358 \\
& & & \\
$\sigma_{\mu}$ & 7.653 & 8.299 & 8.975 \\
$\sigma_{h}$ & 1.092 & 1.076 & 0.840 \\
$\sigma_{\xi}$ & 5.288 & 5.189 & 3.823 \\
\hline & & &
\end{tabular}

Table B.2: Inefficiency factors of the joint return-cash flow model. This table reports the inefficiency factors for the key parameters of the joint return-cash flow model in Section 5.1. For each individual parameter, the inefficiency factor is estimated as $1+2 \sum_{k=1}^{\infty} \rho_{k}$ where $\rho_{k}$ is the $k$ th-order autocorrelation of the chain of retained draws. The estimates use the Newey-West kernel and a bandwidth of $4 \%, 8 \%$, or $15 \%$ of the sample of retained draws. All results are based on a sample of 2,000 retained draws, obtained by sampling a total of 101,000 draws, discarding the first 1,000, and retaining every 20th draw of the post-burn sample. Data: 1973-2016. 